\title{
CRITERION OF ANALYTIC CONTINUABILITY OF FUNCTIONS IN PRINCIPAL INVARIANT SUBSPACES ON CONVEX DOMAINS IN $\mathbb{C}^{n}$
}

\author{
A. S. KRIVOSHEEV
}

\begin{abstract}
Subspaces invariant under differentiation are studied for spaces of functions analytic on domains of a many-dimensional complex space. For a wide class of domains (in particular, for arbitrary bounded convex domains), a criterion of analytic continuability is obtained for functions in arbitrary nontrivial closed principal invariant subspaces admitting spectral synthesis.
\end{abstract}

\section{$\S 1$. Notions AND NOTATiON}

For the reader's convenience, here we collect the main notions used in the paper and fix the notation for them.

$\mathbb{C}^{n}$ is the $n$-dimensional complex space.

$\mathbb{S}$ is the sphere of radius 1 and centered at the origin.

$B(z, \delta)$ is the ball centered at $z=\left(z_{1}, \ldots, z_{n}\right)$ and of radius $\delta$.

$\left\{z_{k}\right\}$ is a sequence of points in $\mathbb{C}^{n}$. The coordinates $z=\left(z_{1}, \ldots, z_{n}\right)$ of a vector $z$ are denoted similarly, but the specific meaning of the symbol $\left\{z_{k}\right\}$ will be clear from the context each time.

For $\varsigma \in \mathbb{S}$ and $a \geq 0$, we denote by $\Phi_{a}(\varsigma)$ the set of sequences $\left\{z_{k}\right\} \subset \mathbb{C}^{n}$ with the following properties:

$$
\text { 1) } \left.\left.\left|z_{k}\right| \rightarrow \infty, k \rightarrow \infty ; \quad 2\right) \lim _{k \rightarrow \infty} z_{k} /\left|z_{k}\right|=\varsigma ; \quad 3\right) \limsup _{k \rightarrow \infty}\left|z_{k+1}\right| /\left|z_{k}\right| \leq 1+a .
$$

Passing to a subsequence, on each sequence $\left\{z_{k}\right\} \in \Phi_{a}(\varsigma)$ we can also impose the additional condition saying that $\left\{\left|z_{k}\right|\right\}$ is monotone decreasing. Indeed, put $k_{1}=1$, and for $j \geq 2$ choose $k_{j}$ to be the minimal $k$ with $\left|z_{k}\right|>\left|z_{k_{j-1}}\right|$. Clearly, $\left\{z_{k_{j}}\right\}$ satisfies 1) and 2). By construction,

$$
\frac{\left|z_{k_{j+1}}\right|}{\left|z_{k_{j}}\right|}=\frac{\left|z_{k_{j+1}}\right|}{\left|z_{k_{j+1}-1}\right|} \frac{\left|z_{k_{j+1}-1}\right|}{\left|z_{k_{j}}\right|} \leq \frac{\left|z_{k_{j+1}}\right|}{\left|z_{k_{j+1}-1}\right|} .
$$

Therefore, $\left\{z_{k_{j}}\right\} \in \Phi_{a}(\varsigma)$.

If $M$ is a subset of $\mathbb{C}^{n}$ and $\varepsilon>0$, we denote by $M[\varepsilon]$ the union of the balls $B(z, \exp (-\varepsilon|z|))$, where $z$ runs through $M$.

$\langle z, \lambda\rangle=z_{1} \lambda_{1}+\cdots+z_{n} \lambda_{n}$.

$\mathbb{Z}_{+}^{n}$ is the set of $n$-vectors with nonnegative integral coordinates.

For $\alpha \in \mathbb{Z}_{+}^{n}$, we put $|\alpha|=\alpha_{1}+\cdots+\alpha_{n}$ and $z^{\alpha}=z_{1}^{\alpha_{1}} \ldots z_{n}^{\alpha_{n}}$.

$H_{M}(z)=\sup _{y \in M} \operatorname{Re}\langle z, y\rangle$ is the support function of a convex set $M \subset \mathbb{C}^{n}$ (more accurately, of the set complex conjugate to $M)$. The function $H_{M}(z)$ is convex and

2010 Mathematics Subject Classification. Primary 46E10, 47B38, 32D15, 32W50.

Key words and phrases. Analytic continuation, invariant subspace, plurisubharmonic function, convex domain. 
positive homogeneous of degree 1 . It is lower semicontinuous and may have $+\infty$ among its values. If $M$ is bounded, $H_{M}(z)$ is bounded and continuous (see [32]).

For a convex domain $D \subset \mathbb{C}^{n}$, we put

$$
\Theta_{D}=\left\{z \in \mathbb{C}^{n}: H_{D}(z)=+\infty\right\} .
$$

If $D$ is bounded, then $\Theta_{D}=\varnothing$. In general, $\Theta_{D}$ may fail to be either open or closed. Since $H_{D}$ is a homogeneous convex function, $\mathbb{C}^{n} \backslash \Theta_{D}$ is a convex cone with vertex at the origin. This cone degenerates to a ray if $D$ is a half-space. The function $H_{D}(z)$ is continuous (see 32] ) in the interior $\operatorname{int}\left(\mathbb{C}^{n} \backslash \Theta_{D}\right)$ of $\mathbb{C}^{n} \backslash \Theta_{D}$.

Let $D$ and $G$ be convex domains in $\mathbb{C}^{n}$ with $D \subset G$. We put

$$
\Xi=\Xi(D, G)=\left\{z \in \mathbb{C}^{n}: H_{D}(z)=H_{G}(z)\right\} .
$$

On the complement of $\Xi(D, G)$ to $\mathbb{C}^{n}$, we have $H_{D}(z)<H_{G}(z)$. In general, $\Xi(D, G)$ may fail to be either open or closed. If $D$ is bounded, $\Xi(D, G)$ is closed.

We put

$$
G(D)=\left\{z \in \mathbb{C}^{n}: \operatorname{Re}\langle z, \xi\rangle<H_{D}(\xi), \xi \in \Xi\right\} .
$$

It is easily seen that $G(D)$ is a convex domain.

For every convex domain $D \in \mathbb{C}^{n}$, we fix a sequence $\left\{K_{m}\right\}$ of compact convex subsets of $D$ that strictly exhaust $D$, i.e., $K_{m} \subset \operatorname{int} K_{m+1}, m=1,2, \ldots$, and $\bigcup K_{m}=D$.

$H(D)$ is the space of functions analytic in a domain $D$ with the topology of uniform convergence on compact subsets of $D$.

$H^{*}(D)$ is the strong conjugate of $H(D)$ (called the space of analytic functionals on $D$ ).

Throughout the paper, the symbol $W$ is used to denote a nontrivial $(W \neq\{0\}$, $W \neq H(D))$ closed subspace of $H(D)$.

$W^{0}$ is the subspace of $H^{*}(D)$ consisting of the functionals that vanish on all elements of $W$. If $W$ is nontrivial, then $W^{0}$ is also nontrivial.

A subspace $W$ is said to be invariant if it is invariant with respect to the operators of partial differentiation, i.e., if along with any function $\varphi(z)$ it contains the derivatives $\partial \varphi / \partial z_{1}, \ldots, \partial \varphi / \partial z_{n}$

Let $\psi(z)$ be a plurisubharmonic function of order (at most) 1 and of finite type (at the order 1 ), i.e., $\psi(z) \leq b+d|z|, z \in \mathbb{C}^{n}$. We denote by $h_{\psi}(z)$ (respectively, $\left.\underline{h}_{\psi}(z)\right)$ its upper (respectively, lower) indicator, that is,

$$
\begin{aligned}
h_{\psi}(z) & =\limsup _{w \rightarrow z} \limsup _{t \rightarrow+\infty} \psi(t w) / t, \\
\underline{h}_{\psi}(z) & =\lim _{\delta \rightarrow 0} \liminf _{t \rightarrow+\infty} \frac{1}{\sigma_{n} \delta^{2 n}} \int_{B(z, \delta)} \frac{\psi(t y)}{t} d \sigma(y),
\end{aligned}
$$

where $d \sigma$ is Lebesgue measure and $\sigma_{n}$ is the volume of the unit ball in $\mathbb{C}^{n}=\mathbb{R}^{2 n}$. If $\psi(z)=\ln |f(z)|$, where $f(z)$ is an entire function of exponential type (that is, $|f(z)| \leq$ $C \exp A|z|, z \in \mathbb{C}^{n}$, for some $A, C>0$ ), in place of $h_{\ln |f|}, \underline{h}_{\ln |f|}$ we write $h_{f}(z)$ and $\underline{h}_{f}(z)$ for simplicity. For $n=1$, the indicator $h_{f}(z)$ is a bounded convex function (consequently, it is continuous); for $n>1$, the function $h_{f}(z)$ is plurisubharmonic but may be discontinuous (see, e.g., 33]).

The definition of the indicators readily implies that they are positive homogeneous of degree 1 and $\underline{h}_{f}(z) \leq h_{f}(z), z \in \mathbb{C}^{n}$. If $\underline{h}_{f}(z)=h_{f}(z)$ for some $z \in \mathbb{C}^{n}$, the function $f$ (more precisely, the plurisubharmonic function $\ln |f|$ ) is said to have (completely) regular growth on the ray $t z, t>0$.

It is well known (see, e.g., [1, Chapter $3, \S 12.7]$ ) that the Laplace transformation $f(z)=$ $(\mu, \exp \langle z, \lambda\rangle)$ for $\mu \in H^{*}(D)$ establishes an isomorphism (algebraically and topologically) between $H^{*}(D)$ and a subspace $P_{D}$ of the space of entire functions of exponential type. 
$P_{D}$ is an inductive limit of Banach spaces, specifically,

$$
\begin{aligned}
& P_{D}=\lim _{m \rightarrow \infty} \operatorname{ind} B_{m}, \\
& B_{m}=\left\{g \in H\left(\mathbb{C}^{n}\right): \sup _{z \in \mathbb{C}^{n}}|g(z)| \exp \left[-H_{K_{m}}(z)\right]<\infty\right\} .
\end{aligned}
$$

If $f \in P_{D}$, the definitions of $h_{f}$ and $P_{D}$ readily show that

$$
h_{f}(z) \leq H_{K_{m}}(z)<H_{D}(z), \quad z \in \mathbb{C}^{n} \backslash\{0\}
$$

for some $m \geq 1$. Conversely, if $h_{f}(z)<H_{D}(z)$ for all $z \in \mathbb{C}^{n} \backslash\{0\}$, then, since $h_{f}$ is upper semicontinuous and $H_{D}$ is lower semicontinuous, for some $m \geq 1$ we have $h_{f}(z) \leq H_{K_{m}}(z), z \in \mathbb{C}^{n}$. Then the Hartogs theorem on the upper limit for families of subharmonic functions (see [1, Chapter 1, Theorem 6.1]) easily implies also the estimate

$$
|f(z)| \leq C \exp H_{K_{m+1}}(z), \quad z \in \mathbb{C}^{n}
$$

(where $C$ is a positive constant). This estimate means that $f \in P_{D}$. Therefore, an entire function $f$ belongs to $P_{D}$ if and only if

$$
h_{f}(z)<H_{D}(z), \quad z \in \mathbb{C}^{n} \backslash\{0\} .
$$

Let $W$ be an invariant subspace of $H(D)$. We denote by $I_{W}$ the subspace of $P_{D}$ that consists of the Laplace transforms of the functionals in $W^{0}$. Let $N(W)$ stand for the set of common zeros of all functions in $I_{W}$, and for $z \in N(W)$, let $m(z)$ be the multiplicity of the zero at $z$; this means that

$$
D^{\alpha} f(z)=\frac{\partial^{|\alpha|} f(z)}{\partial z_{1}^{\alpha_{1}} \cdots \partial z_{n}^{\alpha_{n}}}=0, \quad|\alpha|=0, \ldots, m(z)-1,
$$

for every $z \in N(W)$ and $f_{z} \in I_{W}$, and there exists $f_{z} \in I_{W}$ and a vector $\beta \in \mathbb{Z}_{+}^{n}$ with $D^{\beta} f_{z}(z) \neq 0$.

Let $\Delta(W)$ denote the collection of limit directions of $N(W)$, i.e., the subset of the unit sphere $\mathbb{S}$ consisting of the limits of all convergent sequences of the form $\left\{\lambda_{j} /\left|\lambda_{j}\right|\right\}_{j=1}^{\infty}$, where $\lambda_{j} \in N(W), j=1,2, \ldots$, and $|\lambda(j)| \rightarrow \infty$ as $j \rightarrow \infty$. For a convex domain $D$, we put

$$
D(W)=\left\{z \in \mathbb{C}^{n}: \operatorname{Re}\langle z, \xi\rangle<H_{D}(\xi), \xi \in \Delta(W)\right\} .
$$

If $\Delta(W)=\varnothing$, we agree that $D(W)=\mathbb{C}^{n}$. Clearly, $\Delta(W)$ is a closed set. If $\Delta(W)$ coincides with the sphere $\mathbb{S}$, then the domain $D(W)$ coincides with $D$. In general, $D \subseteq$ $D(W)$, which follows from the representation (see, e.g., 32])

$$
D=\left\{z \in \mathbb{C}^{n}: \operatorname{Re}\langle z, \xi\rangle<H_{D}(\xi), \xi \in \mathbb{S}\right\} .
$$

Put

$$
E(W)=\left\{z^{\alpha} \exp \langle\lambda, z\rangle\right\}_{\lambda \in N(W), 0 \leq|\alpha|<m(\lambda)} .
$$

Then $E(W) \subset W$. Indeed, suppose a function of the form $z^{\alpha} \exp \langle\lambda, z\rangle$ belongs to $E(W)$ but not to $W$. Since $W$ is closed, the Hahn-Banach theorem implies the existence of a function $\mu \in W^{0}$ such that $\left(\mu, z^{\alpha} \exp \langle\lambda, z\rangle\right) \neq 0$. On the other hand, if $f$ is the Laplace transform of $\mu$, the definitions of $N(W)$ and $m(\lambda)$ show that

$$
\left(\mu, z^{\alpha} \exp \langle\lambda, z\rangle\right)=\left(\mu, D^{\alpha}(\exp \langle\lambda, z\rangle)\right)=D^{\alpha}(\mu, \exp \langle\lambda, z\rangle)=D^{\alpha} f(\lambda)=0 .
$$

This contradiction proves the claim.

We say that $W$ admits spectral synthesis if the system $E(W)$ is complete in $W$.

$W$ is called a principal invariant subspace if $N(W)$ is an analytic set of codimension 1 , and moreover, there exists an entire function $F_{W}$ whose zero set coincides with $N(W)$ and $m(\lambda)$ is the multiplicity of its zero $\lambda \in N(W)$. In the case of one variable, all invariant subspaces are principal. It should also be noted that $F_{W}$ is uniquely determined by $W$ up to a factor that is a zero-free entire function. 
Since $N(W)$ is a subset of zeros (with multiplicity taken into account) of entire functions of order 1 belonging to $I_{W}$, this factor can be chosen in such a way that $F_{W}$ will have the first order of growth (and, possibly, an infinite type). In what follows, we assume that, for every principal invariant subspace $W$, some function $F_{W}$ with the above properties is chosen and fixed, and this function is of the first order of growth.

If $G$ is a convex domain containing $D$, we denote by $W(G)$ the closure of the linear span of the system $E(W)$ in the topology of $H(G)$. Along with $W, W(G)$ is a nontrivial closed subspace of $H(G)$ invariant under differentiation, and the definition shows at once that this subspace admits spectral synthesis. If $W$ is principal, then $W(G)$ is also principal.

\section{§2. INTRODUCTION}

In this paper, we study the problem of analytic continuation of all functions in a principal invariant subspace $W \subset H(D)$ to a wider convex domain $G$.

An important example of an invariant subspace is the space of solutions of a homogeneous convolution equation that are analytic in $D$ :

$$
M[\varphi](z)=\mu(\varphi(z+y)) \equiv 0, \quad \varphi \in H(D), \quad \mu \in H^{*}(D) .
$$

The operator $M$ of convolution is well defined because every functional $\mu \in H^{*}(D)$ extends to a continuous linear functional on the space of functions analytic on some compact subset of $D$ (see, e.g., 1, Chapter 3, §14.2]). This operator acts linearly and continuously from $H(D)$ to the space of functions analytic in a neighborhood of the origin and commutes with the differentiation operators. Therefore, the space of solutions of equation (2.1) is closed and invariant under differentiation. The space of solutions of a system of equations such as (2.1) is also closed and invariant under differentiation. It is easily seen that the space $W \subset H(D)$ of solutions of a homogeneous convolution equation is a principal invariant subspace of $H(D)$. Moreover, for the role of $F_{W}$ we can take the Laplace transform of the functional $\mu$ that generates the convolution operator; then $F_{W} \in I_{W}$.

A principal invariant subspace may also coincide with the space of solutions of a system of homogeneous convolution equations. In this case, $F_{W}$ may fail to belong to $I_{W}$; moreover, it may have infinite type at order 1 , and so may fail to be a function of exponential type. Another example of an invariant subspace is provided by the set of functions that expand in a series of exponentials or exponential monomials that converge uniformly on compact subsets of $D$ :

$$
\varphi(z)=\sum_{m=1,|\alpha|=0}^{\infty, k_{m}} c_{m, \alpha} z^{\alpha} \exp \langle\lambda(m), z\rangle, \quad z \in D .
$$

A generalization of this example arises when we consider the closure $W$ in $H(D)$ of the linear span of the system $\left\{p_{\lambda}(z) \exp \langle z, \lambda\rangle, \lambda \in N\right\}$ of exponential polynomials. Here $N$ is a subset of $\mathbb{C}^{n}$. If, for the role of $N$, we take a principal analytic subset in $\mathbb{C}^{n}$ that is part of the zero set of some entire function $f$ of exponential type and, for the role of the $p_{\lambda}(z)$, we take all polynomials whose degree is strictly smaller than the multiplicity $m(\lambda)$ of the zero $\lambda$ of $f$, then we again obtain a principal invariant subspace of $H(D)$.

We list the main problems in the theory of invariant subspaces:

1) spectral synthesis, i.e., approximation of elements of an invariant subspace by exponential polynomials lying in this subspace;

2) the fundamental principle, i.e., representation of functions in an invariant subspace by a series of exponentials, integrals of exponentials, series of integrals of exponentials or exponential polynomials; 
3) analytic continuation of functions belonging to an invariant subspace.

The present paper is devoted to the study of the last question. Originally, this question emerged in a natural way from the problem of extension of convergence for exponential series and for their partial case, power series. The first results pertaining to this question were obtained as early as in the 19th century (see, e.g., 2, 3]). Later, it was observed that the continuation phenomenon is intrinsic not merely for sums of exponential series, but also for more general limits of exponential polynomials. It turned out that functionals analytic in a convex domain and representable there as limits of exponential polynomials may admit analytic continuation to a wider convex domain. Since the closure of exponential polynomials is a basic example of an invariant subspace, the modern statement of the continuation problem looks like this. Under what conditions is it true that all functions in a closed invariant subspace of $H(D)$ that admits spectral synthesis can be continued analytically to a wider convex domain (which must not depend on a particular function). Thus, the continuation problem is stated only for subspaces admitting spectral synthesis. In this connection, it should be noted that, in the case of one variable, the spectral synthesis problem was resolved completely in [4] and [5]. In the case of several variables, it is known that the space of solutions of a homogeneous convolution equation in an arbitrary convex domain admits spectral synthesis; see [6, 7].

As to the continuation problem, it has a long history. See 8 for a detailed survey of the relevant results. Here we only remark that, in the case of one variable, the continuation problem has been studied in [9] -24 and elsewhere. For $n=1$, its complete solution was found in 25] and 26].

In the case of several variables, the question has been treated in [27]- 31, [7, 24, 8] and elsewhere. For $n>1$, only certain conditions sufficient for continuability have been obtained. No necessary conditions have been found. Moreover, only continuation to the maximal possible convex domain has been considered. Moreover, only the particular case has been treated in which $W \subset H(D)$ is the space of solutions of the homogeneous convolution equation (2.1), and $D$ is the algebraic sum of another convex domain $D^{\prime}$ and a compact set $K$ such that the support function $H_{K}(z)$ coincides with the upper indicator $h_{f}(z)$ of the Laplace transform $f(z)$ for the functional $\mu$ generating the convolution operator. Sufficient conditions have been stated in terms of various lower estimates for $f(z)$. For example, the regularity of growth of the entire function $f(z)$ of exponential type was assumed on some subset of $\mathbb{C}^{n}$ (see [30]), or some lower estimates were imposed on $\ln |f(z)|$ at the points of a surface that bounds the zero set of $f(z)$ (this set coincides with $N(W)$ ) in the case in question) and situated at a fairly small distance from $N(W)$ (see [8]). Other sorts of estimates have also occurred; see [28, 29, 31].

In the present paper, an essentially novel method for solution of the continuation problem is developed. In distinction to earlier papers, it is based on plurisubharmonic rather than on entire functions. The passage from entire functions to "softer" objects (such as plurisubharmonic functions) has made it possible to obtain a continuability criterion for arbitrary principal invariant subspaces admitting spectral synthesis in arbitrary convex domains in $\mathbb{C}^{n}$ subject to a certain natural condition when the domain is unbounded. In the case of a bounded domain, the continuation problem is solved completely. Moreover, a criterion is obtained for an arbitrary domain that contains the initial domain and is included in the maximal possible one. All results on continuation obtained earlier in the case of one variable, as well as all results for many variables and pertaining to principal invariant subspaces, are included in the result of the present paper as partial cases. This means that only the results of [24] are left apart.

We briefly describe the layout of the paper. In $\S 3$, we reduce the continuation problem to the dual problem of the theory of functions of exponential type, which may be treated 
as a problem of division "with residual" in the space of entire functions. On this basis, also in $\S 3$, we describe the maximal possible convex domain to which all functions in $W \subset H(D)$ may happen to be continuable.

In $\S 4$, conditions sufficient for continuability are presented. They are stated in terms of lower estimates for certain plurisubharmonic functions on surfaces that bound the set $N(W)$.

In $\S 5$ and $\S 6$, some auxiliary results are collected (some of which are of independent interest) about the construction of special plurisubharmonic functions and the geometry of convex domains. These results will be employed to obtain necessary conditions and, eventually, the criterion of continuability.

In $\S 7$, conditions necessary for continuability are presented. Like sufficient conditions, they are stated in terms of lower estimates for plurisubharmonic functions, but the estimates are local this time.

In $\S 8$, the necessary and sufficient conditions are combined to yield a continuability criterion. Several reformulations of this criterion are presented; all of them involve estimates of plurisubharmonic functions. These statements are quite convenient for applications (in particular, for obtaining further restatements) because they impose minimal possible restrictions. However, their form is somewhat unusual and the perception of them presents some difficulties.

In this connection, in $\S 10$ we present yet another series of equivalent reformulations of the continuability criterion, which are of simple form and involve, in particular, the notion of regular growth for entire functions. This notion is used in the solution of many problems in the theory of analytic, entire, or plurisubharmonic functions and in the theory of convolution operators and invariant subspaces. A series of classical problems have been resolved up to now in terms of regular growth. Among them, we can name the representability of functions analytic in convex domains of the complex plane by series of exponentials, the fundamental principle for invariant subspaces, interpolation in spaces of entire functions, solvability of nonhomogeneous convolution equations and of systems of such equations, etc. This notion is used so widely because of the rigid relationship between the regularity of growth of an entire function and the distribution of its zeros (see 40, 37). Thus, resolving a problem in terms of regular growth, we obtain a solution in terms of the geometry of the zero set of the entire function.

In order to pass from plurisubharmonic to entire functions, in $\S 9$ we establish a special type of approximability of plurisubharmonic functions by logarithms of the moduli of entire functions. This result is based on Yulmukhametov's well-known theorem (see [39]).

Finally, in $\S 11$, some general examples of invariant subspaces are presented (in particular, we treat results of forerunners as examples), and certain specific invariant subspaces are treated in the light of the continuability problem.

To finish the introduction, I find it pertinent to give a concise formulation of the problem to be resolved here. Let $W \subset H(D)$ be a nontrivial closed principal invariant subspace admitting spectral synthesis. Find conditions ensuring the analytic continuability of every function in $W$ to some domain $G \supset D$. What is the class of admissible domains $G$ ?

\section{§3. THE DUAL PROBLEM}

In this section, we state and prove a result that reduces the continuability problem to "division with residual" in the theory of entire functions. This result has been used in some form in the greater part of papers on continuability. We start with an auxiliary statement. 
Lemma 3.1. Let $D$ be a convex domain in $\mathbb{C}^{n}$ and $W$ an invariant subspace of $H(D)$. The following statements are equivalent.

1) $W$ admits spectral synthesis;

2 ) the set $I_{W}$ consists precisely of the functions $f \in P_{D}$ that have zeros (at least) at the points $\lambda \in N(W)$ with multiplicity at least $m(\lambda)$.

Proof. 1) $\Rightarrow 2$ ). Let $f \in I_{W}$. The definition of $I_{W}$ shows that $f \in P_{D}$, and the definitions of $N(W)$ and $m(\lambda)$ show that $f$ has a zero of multiplicity at least $m(\lambda)$ at every $\lambda \in N(W)$. Conversely, if $f$ belongs to $P_{D}$, it is the Laplace transform of some functional $\mu \in H^{*}(D)$. If, moreover, $f$ has a zero of multiplicity at least $m(\lambda)$ at every $\lambda \in N(W)$, the definition of a Laplace transform shows easily that $\mu$ vanishes at all elements of the system $E(W)$. The space $W$ admits spectral synthesis; that is, $E(W)$ is complete in $W$. Consequently, $\mu$ vanishes on $W$, i.e., belongs to $W^{0}$. Thus, its Laplace transform belongs to $I_{W}$.

$2) \Rightarrow 1)$. Let $\mu$ be an arbitrary functional in $H^{*}(D)$ that vanishes on the elements of the system $E(W)$, and let $f$ be its Laplace transform. Then $f$ belongs to $P_{D}$ and has a zero of multiplicity at least $\lambda \in N(W)$ at every $\lambda \in L(W)$. By 2), we have $f \in I_{W}$. By the Hahn-Banach theorem, this implies that the system $E(W)$ is complete in $W$.

Remark. Lemma 3.1 shows that if $W$ is a principal invariant subspace, then $I_{W}$ consists precisely of the functions $f \in P_{D}$ that are divisible by $F_{W}$.

Now, we show how continuability is reduced to "division with residual" for entire functions. Most of the papers on continuability have employed some form of this result.

Lemma 3.2. Let $D$ and $G$ be convex domains in $\mathbb{C}^{n}$, and let $D \subset G$. Suppose $W \subset$ $H(D)$ is an invariant subspace admitting spectral synthesis. The following statements are equivalent.

1) Every function in $W$ admits analytic continuation to $G$ and can be approximated by linear combinations of elements of $E(W)$ on $G$.

2) For every $h \in P_{G}$ there exist $f \in I_{W(G)}$ and $g \in P_{D}$ such that $h=f+g$.

Proof. By the definition of $W(G)$ and the completeness of the system $E(W)$ in $W$, the restriction operator $R$ takes $W(G)$ to $W$. Clearly, this operator is linear and continuous. By the uniqueness theorem for analytic functions, it is injective. Statement 1) is equivalent to its surjectivity, consequently, to bijectivity. Since $W(G)$ and $W$ are closed subspaces of the Fréchet spaces $H(G)$ and $H(D)$ (see, e.g., [1]), this is equivalent to the statement that $R^{*}: W^{*} \rightarrow W^{*}(G)$ is a bijection (see [34, Chapter 8, $\left.\S 6.18\right]$ ). $H(D)$ and $H(G)$ are also Schwartz spaces. Therefore, we have isomorphisms (see [35])

$$
W^{*} \cong H^{*}(D) / W^{0}, \quad W^{*}(G) \cong H^{*}(G) / W^{0}(G)
$$

Thus, 1) is equivalent to the statement that for every $\nu \in H^{*}(G)$ there is a unique equivalence class $[\mu] \in H^{*}(D) / W^{0}$ such that

$$
(\nu, \varphi)=(\mu, R(\varphi)), \quad \varphi \in W(G) .
$$

Since the functionals $\nu$ and $\mu$ are continuous and the system $E(W)$ is complete, we may restrict ourselves to $\varphi \in E(W)$ in this relation. Let $h(\lambda)$ and $g(\lambda)$ be the Laplace transforms of $\nu$ and $\mu$. By the above, we have

$$
D^{\alpha} h(\lambda)=\left(\nu, z^{\alpha} \exp \langle\lambda, z\rangle\right)=\left(\mu, z^{\alpha} \exp \langle\lambda, z\rangle\right)=D^{\alpha} g(\lambda), \quad \lambda \in N(W), \quad 0 \leq|\alpha|<m(\lambda) .
$$

Thus, 1) is equivalent to the statement that for every $h \in P_{G}$ there is a unique equivalence class $[g] \in P_{D} / I_{W}$ such that $h-g$ vanishes at every $\lambda \in N(W)$ with multiplicity at least 
$m(\lambda)$, i.e., $(h-g) \in I_{W(G)}$. To complete the proof, it remains to observe that if $h=f+g$ and simultaneously $h=f_{1}+g_{1}$, then

$$
\left(g-g_{1}\right)=\left(-f+f_{1}\right) \in I_{W(G)} .
$$

This implies that $\left(g-g_{1}\right) \in I_{W}$ because $\left(g-g_{1}\right) \in P_{D}$. Therefore, $g$ and $g_{1}$ represent the same equivalence class in $P_{D} / I_{W}$.

The next result characterizes the maximal convex domain to which all functions in $W$ may extend. As a preliminary, we observe that, since $D \subseteq D(W)$, we always have

$$
H_{D}(\xi) \leq H_{D(W)}(\xi), \quad \xi \in \mathbb{C}^{n} .
$$

On the other hand, the definition of $D(W)$ readily shows that

$$
H_{D(W)}(\xi) \leq H_{D}(\xi), \quad \xi \in \Delta\left(F_{W}\right) .
$$

Thus, we have the identity

$$
H_{D(W)}(\xi)=H_{D}(\xi), \quad \xi \in \Delta\left(F_{W}\right) .
$$

Lemma 3.3. Let $D$ be a convex domain in $\mathbb{C}^{n}$ and $W \subset H(D)$ an invariant subspace admitting spectral synthesis. Suppose that all functions in $W$ admit analytic continuation to a convex domain $G \supset D$ and, on $G$, can be approximated by linear combinations of elements of $E(W)$. Then $G \subseteq D(W)$.

Proof. By Lemma 3.2, for every $h \in P_{G}$ there exists $g \in P_{D}$ with $(h-g) \in I_{W(G)}$. By the definition of $P_{D}$, there is an index $m \geq 1$ and $c>0$ such that

$$
|g(\lambda)| \leq c \exp H_{K_{m}}(\lambda), \quad \lambda \in \mathbb{C}^{n} .
$$

Since $h(\lambda)$ and $g(\lambda)$ coincide on $N(W)$, it follows that

$$
|h(\lambda)| \leq c \exp H_{K_{m}}(\lambda), \quad \lambda \in N(W) .
$$

Suppose $G$ is not included in $D(W)$. By the definition of $D(W)$, for some $z_{0} \in G$ and $\lambda_{0} \in \Delta\left(F_{W}\right)$ we have

$$
\operatorname{Re}\left\langle z_{0}, \lambda_{0}\right\rangle \geq H_{D}\left(\lambda_{0}\right) .
$$

By the choice of $z_{0}$, the function $\exp \left\langle z_{0}, \lambda\right\rangle$ belongs to $P_{G}$. Therefore, it satisfies (3.2). On the other hand, the last inequality, the embedding $K_{m} \subset D$, and the continuity of the function $H_{K_{m}}(\lambda)$ imply the existence of $c, \delta>0$ such that

$$
\left|\exp \left\langle z_{0}, \lambda\right\rangle\right| \geq \exp \left\{H_{K_{m}}(\lambda)+\varepsilon|\lambda|\right\}, \quad H_{K_{m}}(\lambda) \in B\left(\lambda_{0}, \delta\right) .
$$

Using the definition of $\Delta(W)$, we choose a sequence $\lambda_{j} \in N(W), j \geq 1$, such that $\left|\lambda_{j}\right| \rightarrow \infty$ and $\frac{\lambda_{j}}{\left|\lambda_{j}\right|}$ as $j \rightarrow \infty$. Since the function $H_{K_{m}}(\lambda)$ is homogeneous, the last inequality is true at the point $\lambda=\lambda_{j}$ for all sufficiently large $j$. This contradicts (3.2).

\section{$\S 4$. Conditions Sufficient FOR COntinuability}

Before stating the conditions mentioned in the title of the section, we prove two auxiliary statements. By Lemma 3.3, all functions in $W$ admit analytic continuation to a convex domain $G \supset D$ only if $G$ is a subdomain of $D(W)$. Moreover, by Lemma 3.2 and the remark to Lemma 3.1, in the case of a principal invariant subspace this type of continuability is equivalent to the existence of a function $g \in P_{D}$ (that depends on $\left.h \in P_{G}\right)$ such that $h-g$ is divisible by $F_{W}$. In order to prove the existence of $g$, we shall need a special cutoff function, which will be constructed in the next lemma. 
Lemma 4.1. Suppose $M$ is a subset of $\mathbb{C}^{n}$ and $\varepsilon>0$. There exists a function $E_{M} \in$ $C^{\infty}\left(\mathbb{C}^{n}\right)$ with the following properties:

1) $0 \leq E_{M}(z) \leq 1, z \in \mathbb{C}^{n}$;

2) $E_{M}(z)=1, z \in M$

3) $E_{M}(z)=0, z \notin M[\varepsilon]$

4) $\left|\partial E_{M}(z) / \partial \bar{z}_{i}\right| \leq C \exp (\varepsilon(|z|+2)), z \in \mathbb{C}^{n}, i=1, \ldots, n$ (the constant $C$ depends on the dimension only).

Proof. Put

$$
M_{k}=M \cap\{z: k-1 \leq|z| \leq k\}, \quad k=1,2, \ldots, \quad \Omega_{k}=M_{k}+B(0, \exp (-\varepsilon k)) .
$$

As usual (see, e.g., [36. Theorem 1.4.1 and formula (1.4.2)]), we can find functions $e_{k} \in$ $C^{\infty}\left(\mathbb{C}^{n}\right), k=1,2, \ldots$, such that

$$
\begin{aligned}
& 0 \leq e_{k}(z) \leq 1, \quad z \in \mathbb{C}^{n} ; \quad e_{k}(z)=1, \quad z \in M_{k} ; \quad e_{k}(z)=0, \quad z \notin \Omega_{k} ; \\
& \left|\partial e_{k}(z) / \partial \bar{z}_{i}\right| \leq c \exp (\varepsilon k), \quad z \in \mathbb{C}^{n}, \quad i=1, \ldots, n,
\end{aligned}
$$

where $c>0$ depends only on the dimension $n$. Put

$$
E_{M}(z)=1-\prod_{k=1}^{\infty}\left(1-e_{k}(z)\right) .
$$

The function $E_{M}(z)$ is well defined, because every $z \in \mathbb{C}^{n}$ belongs to at most 3 sets among the $\Omega_{k}, k=1,2, \ldots$, whence we see that at most 3 factors in the product are different from 1 . We show that this function satisfies the required conditions. Condition 1) for $E_{M}(z)$ is fulfilled because it is fulfilled for the $e_{k}(z), k=1,2, \ldots$ Let $z \in M$. Then $z \in M_{k}$ for some $k \geq 1$. Therefore, $e_{k}(z)=1$ and $E_{M}(z)=1$. This yields 2). By the definition of $E_{M}(z)$ and the properties of the $e_{k}(z), k=1,2, \ldots$, we shall verify condition 3) if we show that $\Omega_{k} \subset M[\varepsilon], k=1,2, \ldots$ Let $z \in \Omega_{k}$. Take $y \in M_{k}$ with $z \in B(y, \exp (-\varepsilon k))$. The definition of $M_{k}$ shows that $|y-z|<\exp (-\varepsilon k) \leq \exp (-\varepsilon|y|)$. Thus, $z \in M[\varepsilon]$. Again let $z \in \Omega_{k}$. Then $|z|+2 \geq k$. By the estimates for the derivatives of $e_{k}$ and the relation $e_{k}(w)=0, w \notin \Omega_{k}$, we have

$$
\left|\partial e_{k}(w) / \partial \bar{w}_{i}\right| \leq c \exp (\varepsilon(2+|w|)), \quad w \in \mathbb{C}^{n}, i=1, \ldots, n .
$$

Consequently,

$$
\left|\partial E_{M}(w) / \partial \bar{w}_{i}\right| \leq C \exp (\varepsilon(2+|w|)), \quad w \in \mathbb{C}^{n}, \quad i=1, \ldots, n,
$$

where $C=3 c$.

Before passing to the main result of the section, we prove another technical statement.

Lemma 4.2. Let $q(z)$ be a positive homogeneous function of degree 1 that is continuous on $\mathbb{C}^{n}$. For every $\beta>0$ there exists $\delta>0$ such that

$$
\sup _{\lambda \in B(z, \delta|z|)} q(\lambda) \leq \inf _{\lambda \in B(z, \delta|z|)} q(\lambda)+\beta|z|, \quad z \in \mathbb{C}^{n} .
$$

Proof. We fix $\beta>0$. Since $q(z)$ is uniformly continuous on the sphere $\mathbb{S}$, there exists $\delta>0$ such that for arbitrary $z \in \mathbb{S}$ and all $\lambda, w \in B(z, \delta)$ we have

$$
|q(\lambda)-q(w)| \leq \beta .
$$

Since $q$ is homogeneous, it follows that

$$
\sup _{\lambda \in B(z, \delta|z|)} q(\lambda)=|z| \sup _{\lambda \in B(z /|z|, \delta)} q(\lambda) \leq|z| \inf _{\lambda \in B(z /|z|, \delta)}(q(\lambda)+\beta)=\inf _{\lambda \in B(z, \delta|z|)} q(\lambda)+\beta|z| .
$$


Now we turn to the main topic of this section: conditions sufficient for the continuability of functions in principal invariant subspaces.

Theorem 4.1. Let $D$ be a convex domain in $\mathbb{C}^{n}$, and let $W$ be a principal invariant subspace of $H(D)$ admitting spectral synthesis. Let $G$ be a convex subdomain of $D(W)$ containing D. Suppose that every neighborhood $V$ of $\Xi \cap \mathbb{S}$ includes a compact set $X \subseteq \mathbb{S}$ with the following property: for every index $m$ and every $\varepsilon>0$, there is a function $\psi(z)$ plurisubharmonic in $\mathbb{C}^{n}$, a number $R>0$, an index $p$, and an open set $U \subset \mathbb{C}^{n}$ such that

i) $N(W) \subset U$;

ii) for every $z \in U[\varepsilon] \backslash B(0, R)$, the point $z /|z|$ belongs to $X$;

iii) $\psi(z)+\ln \left|F_{W}(z)\right| \geq H_{K_{m}}(z), z \in U[\varepsilon] \backslash U$;

iv) $\psi(z)+\ln \left|F_{W}(z)\right| \leq H_{K_{p}}(z), z \neq B(0, R)$.

Then every function in $W$ admits analytic continuation to $G$ and can be approximated on $G$ by linear combinations of elements of $E(W)$.

Remark. If the set $\Xi \cap \mathbb{S}$ is closed (this is the case, for instance, provided $D$ is bounded), we may restrict ourselves to $V$ running through some countable fundamental system of neighborhoods of this set. The role of $U$ will every time be played by the union of the cone with vertex at the origin that is generated by some of these neighborhoods, and a ball of sufficiently large radius. In this setting, there is no need to introduce the compact set $X$. Moreover, condition i) becomes redundant because it is fulfilled automatically (this follows from the definition of $\Delta(W)$ and the inclusion $\Delta(W) \subset \Xi$; the latter is true by (3.1)). Condition ii) also becomes redundant. Since $\left\{K_{m}\right\}$ is an increasing sequence of compact subsets of $D$, conditions iii) and iv) imply the inequalities $H_{K_{m}}(z) \leq H_{K_{p}}(z)<H_{D}(z)$. Next, the sequence $\left\{K_{m}\right\}$ exhausts the domain $D$. Thus, if $m$ is sufficiently large, the gap between these functions will be arbitrarily small on $\mathbb{S}$ (provided $D$ is bounded). Therefore, conditions iii) and iv) mean that the function $H_{D}(z)-\ln \left|F_{W}(z)\right|$ can be approximated "from below" by functions plurisubharmonic in $\mathbb{C}^{n}$. In fact, it suffices to ensure approximation only on $U[\varepsilon] \backslash U$. This set is a "thin" one-sided neighborhood of $\partial U$ containing the zero set $N(W)$.

Proof. As has already been mentioned, the claim will be proved if we show that for every $h \in P_{G}$ there exists $g \in P_{D}$ such that $h-g$ is divisible by $F_{W}$. In other words, given $h$, we must find a function $g$ with smaller growth estimates that coincides with $h$ at every $z \in N(W)$ together with all its partial derivatives up to order $m(z)-1$ (inclusive). At the first stage, by using Lemma 4.1, we shall construct such a function analytic only in a special neighborhood of $N(W)$.

So, we fix an arbitrary function $h \in P_{G}$. By the definition of $P_{G}$, we can find a compact convex set $K \subset G$ and a constant $c>0$ with

$$
|h(z)| \leq c \exp \left(H_{K}(z)\right), \quad z \in \mathbb{C}^{n} .
$$

Since $K$ is compactly embedded in $G$, we have

$$
H_{K}(z)<H_{G}(z), \quad z \in \mathbb{C}^{n} \backslash\{0\} .
$$

The definition of the set $\Xi$ shows that

$$
H_{K}(z)<H_{D}(z), \quad z \in \Xi \backslash\{0\} .
$$

Since $H_{K}$ is continuous and $H_{D}$ is lower semicontinuous, this inequality remains true in some neighborhood $V$ of $\Xi \cap \mathbb{S}$. We find a compact set $X \subset V$ as described in the assumptions of the theorem. The last inequality and the definition of the support function imply that for every $z \in X$ there exists $w(z) \in D$ such that

$$
H_{K}(z)<\operatorname{Re}\langle w(z), z\rangle .
$$


By continuity, this inequality extends to a neighborhood $\Omega(z)$ of $z$. The sets $\Omega(z)$, $z \in X$, cover $X$, and we can extract a finite subcovering $\Omega\left(z_{1}\right), \ldots, \Omega\left(z_{j}\right)$. Since the $K_{m}, m=1,2, \ldots$, exhaust $D$, there exists an index $s$ such that $K_{s}$ contains all of $w\left(z_{1}\right), \ldots, w\left(z_{j}\right)$. Consequently,

$$
H_{K}(z)<H_{K_{s}}(z), \quad z \in X
$$

By (4.1) and the homogeneity of a support function, it follows that

$$
|h(t z)| \leq c \exp \left(H_{K_{s}}(t z)\right), \quad z \in X, \quad t \geq 0 .
$$

Since $K_{s}$ lies in the interior of $K_{s+1}$, there exists $\varepsilon>0$ such that

$$
H_{K_{s}}(z)+\varepsilon|z| \leq H_{K_{s+1}}(z), \quad z \in \mathbb{C}^{n} .
$$

By the assumptions of the theorem, for the index $m=s+1$ and the number $\varepsilon$ there exists a plurisubharmonic function $\psi(z)$ on $\mathbb{C}^{n}$, a number $R>0$, an index $p$, and an open set $U$ satisfying i)-iv). Let $E_{U}(z)$ be the function constructed in Lemma 4 for these $U$ and $\varepsilon>0$. We put $\varphi(z)=E_{U}(z) h(z)$. Then $\varphi$ is infinitely differentiable, and by property 2) of the function $E_{U}(z)$ and condition i), the functions $h$ and $\varphi$ coincide in the neighborhood $U$ of $N(W)$. In particular, this means that

$$
D^{\alpha} h(z)=D^{\alpha} \varphi(z), \quad z \in N(W),|\alpha|=0,1, \ldots, m(z)-1 .
$$

Moreover, properties 1) and 2) of $E_{U}(z)$, condition ii), and inequality (4.2) imply the estimate

$$
|\varphi(z)| \leq c_{1} \exp \left(H_{K_{s}}(z)\right), \quad z \in \mathbb{C}^{n},
$$

where $c_{1}$ is a positive constant.

In order to construct the required function $g \in P_{D}$, it remains to "correct" $\varphi$ to obtain an entire function with due growth estimate that still satisfies (4.4). We shall do this by adding to $\varphi$ an infinitely differentiable function that vanishes at every $z \in N(W)$ with multiplicity at least $m(z)$. So, we seek $g$ in the form $g=\varphi+F_{W} \eta$, where the function $\eta$ is to be determined. Since $g$ must be entire, we should look for $\eta$ among the solutions of the equation $0=\bar{\partial} g=\bar{\partial} \varphi+F_{W} \bar{\partial} \eta$, or

$$
\bar{\partial} \eta=-\bar{\partial} \varphi / F_{W}
$$

This solution should obey an appropriate estimate. To prove the existence of such a solution, first we must estimate the right-hand side of (4.6). To begin with, we observe that, by property 3 ) of $E_{U}(z)$, the function $\varphi$ (and, with it, $\bar{\partial} \varphi$ ) vanishes outside $U[\varepsilon]$. Next, as has already been mentioned, $h$ and $\varphi$ coincide on $U$. Since $h$ is analytic, we see that $\bar{\partial} \varphi(z)=0, z \in U$. Now, using consecutively property 4) of $E_{U}(z)$, condition ii) of the theorem, and inequalities (4.2) and (4.3), for every $z \in U[\varepsilon]$ we obtain

$$
\begin{aligned}
\|\bar{\partial} \varphi(z)\|^{2} & =\left\|\bar{\partial} E_{U}(z) h(z)\right\|^{2}=|h(z)|^{2} \sum_{j=1}^{n}\left|\frac{\partial E_{U}(z)}{\partial \bar{z}_{j}}\right|^{2} \\
& \leq c_{2} \exp 2\left(H_{K_{s}}(z)+\varepsilon|z|\right) \leq c_{2} \exp 2 H_{K_{s+1}}(z)=c_{2} \exp 2 H_{K_{m}}(z),
\end{aligned}
$$

where $c_{2}$ is a positive constant. Together with iii), this implies that

$$
\left\|\bar{\partial} \varphi(z) / F_{W}(z)\right\|^{2} \leq c_{2} \exp 2 \psi(z), \quad z \in U[\varepsilon] \backslash U .
$$

Therefore, taking into account the above information about $\bar{\partial} \varphi$, we arrive at

$$
\left\|\bar{\partial} \varphi(z) / F_{W}(z)\right\|^{2} \leq c_{2} \exp 2 \psi(z), \quad z \in \mathbb{C}^{n} .
$$


This estimate means that for every $\tau>0$ we have

$$
\int_{\mathbb{C}^{n}}\left\|\frac{\bar{\partial} \varphi(z)}{F_{W}(z)}\right\|^{2} \exp 2(-\psi(z)-\tau|z|) d \sigma(z)=B<\infty .
$$

Then (see, e.g., 33, Chapter 3, §6, Theorem 3.6.2]), in the space of functions on $\mathbb{C}^{n}$ with locally integrable square of the modulus, there is an element $\eta$ satisfying (4.6) (as a distribution) and obeying the inequality

$$
\int_{\mathbb{C}^{n}}|\eta(z)|^{2} \exp 2(-\psi(z)-\tau|z|)\left(1+|z|^{2}\right)^{-2} d \sigma(z) \leq 2^{-1} B .
$$

We put $g=\varphi+F_{W} \eta$. By (4.6), the distributional derivatives of $g$ by $\bar{z}_{j}, j=1, \ldots, n$, vanish on $\mathbb{C}^{n}$. It is well known that then $g$ is analytic on $\mathbb{C}^{n}$. We show that $g \in P_{D}$. By the mean value inequality for subharmonic functions, we have

$$
|g(z)| \leq \frac{1}{\sigma_{n}} \int_{B(z, 1)}|g(\lambda)| d \sigma(\lambda), \quad z \in \mathbb{C}^{n}
$$

By (4.5) and the Cauchy-Bunyakovskir inequality, we deduce that

$$
\begin{aligned}
|g(z)| \leq & \left(\sigma_{n}\right)^{-1} \int_{B(z, 1)}\left|\varphi(\lambda)+F_{W}(\lambda) \eta(\lambda)\right| d \sigma(\lambda) \\
\leq & \left(\sigma_{n}\right)^{-1} \int_{B(z, 1)}|\varphi(\lambda)| d \sigma(\lambda)+\left(\sigma_{n}\right)^{-1} \int_{B(z, 1)}\left|F_{W}(\lambda) \eta(\lambda)\right| d \sigma(\lambda) \\
\leq & c_{1} \exp \left(\sup _{\lambda \in B(z, 1)} H_{K_{s}}(\lambda)\right) \\
& +\left(\sigma_{n}\right)^{-1}\left(\int_{B(z, 1)}\left|F_{W}(\lambda) \eta(\lambda)\right|^{2} \exp 2\left(-H_{K_{p}}(\lambda)-2 \tau|\lambda|\right) d \sigma(\lambda)\right)^{1 / 2} \\
& \times\left(\int_{B(z, 1)} \exp 2\left(H_{K_{p}}(\lambda)+2 \tau|\lambda|\right) d \sigma(\lambda)\right)^{1 / 2}, \quad z \in \mathbb{C}^{n} .
\end{aligned}
$$

Lemma 4.2 and inequality (4.3) imply the existence of a constant $c_{3}>0$ such that

$$
c_{1} \exp \left(\sup _{\lambda \in B(z, 1)} H_{K_{s}}(\lambda)\right) \leq c_{3} \exp H_{K_{s+1}}(z)=c_{3} \exp H_{K_{m}}(z) \quad \text { for all } z .
$$

Next, again by Lemma 4.2, we obtain

$$
\begin{aligned}
\left(\int_{B(z, 1)} \exp 2\left(H_{K_{p}}(\lambda)+2 \tau|\lambda|\right) d \sigma(\lambda)\right)^{1 / 2} & \leq\left(\left(\sigma_{n}\right) \exp \left(2 \sup _{\lambda \in B(z, 1)}\left(H_{K_{p}}(\lambda)+2 \tau|\lambda|\right)\right)\right)^{1 / 2} \\
& \leq c_{4}\left(\sigma_{n}\right)^{1 / 2} \exp \left(H_{K_{p}}(z)+3 \tau|z|\right), \quad z \in \mathbb{C}^{n}
\end{aligned}
$$

where $c_{4}$ is a positive constant. Finally, condition iv) in the theorem and estimate (4.7) imply the inequality

$$
\left(\int_{B(z, 1)}\left|F_{W}(\lambda) \eta(\lambda)\right|^{2} \exp 2\left(-H_{K_{p}}(\lambda)-2 \tau|\lambda|\right) d \sigma(\lambda)\right)^{1 / 2} \leq\left(B^{\prime}\right)^{1 / 2}
$$

for some $B^{\prime} \geq B / 2$. Taking (4.8)-(4.10) into account, we obtain

$$
\begin{aligned}
|g(z)| & \leq c_{3} \exp H_{K_{m}}(z)+c_{4}\left(B^{\prime}\left(\sigma_{n}\right)^{-1}\right)^{1 / 2} \exp \left(H_{K_{p}}(z)+3 \tau|z|\right) \\
& \leq c_{5} \exp \left(H_{K_{p}}(z)+3 \tau|z|\right), \quad z \in \mathbb{C}^{n} .
\end{aligned}
$$


The last inequality follows from conditions iii) and iv) and the embeddings $K_{j} \subset K_{j+1}$, $j=1,2, \ldots$. Since $K_{p}$ lies in the interior of $K_{p+1}$, for sufficiently small $\tau>0$ we have

$$
H_{K_{p}}(z)+3 \tau|z| \leq H_{K_{p+1}}(z), \quad z \in \mathbb{C}^{n} .
$$

Thus, we arrive at

$$
|g(z)| \leq c_{5} \exp H_{K_{p+1}}(z), \quad z \in \mathbb{C}^{n},
$$

which means that $g \in P_{D}$. It remains to prove that $h-g$ is divisible by $F_{W}$. For this, we recall that $\bar{\partial} \varphi(z)=0, z \in U$. Consequently, by (4.6), the function $\eta(z)$ is analytic in the neighborhood $U$ of $N(W)$. Therefore,

$$
D^{\alpha}\left(F_{W}(z) \eta(z)\right)=0, \quad z \in N(W),|\alpha|=0,1, \ldots, m(z)-1 .
$$

Together with (4.4), this implies that

$$
D^{\alpha}(h(z)-g(z))=0, \quad z \in N(W),|\alpha|=0,1, \ldots, m(z)-1 .
$$

The last formula means that $(h-g) / F_{W}$ is an entire function.

\section{§5. Construction of plurisubharmonic functions}

For obtaining necessary conditions and, eventually, a continuability criterion, we need some special plurisubharmonic functions. We begin with some auxiliary results. The following three statements are refinements and generalizations of some known results about lower estimates for the modulus of an analytic function, and about division for entire functions of finite order and type.

Lemma 5.1. Let $f(z)$ be a function nonconstant and analytic in the ball $B\left(z_{0}, 2 e R\right)$, such that $f\left(z_{0}\right)=1$ and

$$
\ln |f(z)| \leq M, \quad z \in B\left(z_{0}, 2 e R\right) .
$$

Then for every $\eta \in(0,1 / 9), r \in(0,(1-9 \eta) R)$, and $\varsigma \in \mathbb{S}$ there exists $\tau \in(r, r+9 \eta R)$ such that

$$
\ln \left|f\left(z_{0}+\lambda \varsigma\right)\right| \geq-(4-\ln \eta) M, \quad|\lambda|=\tau .
$$

Proof. First, we note that $M>0$ by the maximum of the modulus principle. Fix $\eta \in(0,1 / 9), r \in(0,(1-9 \eta) R)$, and $\varsigma \in \mathbb{S}$. Consider the function $g(\lambda)=f\left(z_{0}+\lambda \varsigma\right)$, $\lambda \in B(0,2 e R)$. By the lower estimate for the modulus of a function analytic in a disk (see [38, Theorem 4.2]), we have

$$
\ln |g(\lambda)| \geq-(2+\ln (3 e / 2 \eta)) M \geq-(4-\ln \eta) M
$$

inside the disk $|\lambda| \leq R$ but outside some exceptional disks whose radii have a sum of $4 \eta R$. The circular projections of the exceptional disks to the positive real semiaxis have lengths of at most $8 \eta R$; therefore, in the annulus $r<|\lambda|<r+9 \eta R$ there is a circle $|\lambda|=\tau$ that does not intersect these disks. Thus, (5.1) is fulfilled at each point of this circle.

Lemma 5.2. Let $F$ be an entire function of order (at most) $\rho$ and of finite type (at the order $\rho$ ), and let $\psi$ be a plurisubharmonic function such that $\psi+\ln |F|$ is also of order (at most) $\rho$ and of finite type (at the order $\rho$ ), i.e.,

$$
\psi(z)+\ln |f(z)| \leq A+B|z|^{\rho}, \quad \ln |F(z)| \leq C+D|z|^{\rho}, \quad z \in \mathbb{C}^{n} .
$$

Then

$$
\psi(z) \leq A+7 C-8 \ln |F(\xi)|+(7 D+B) 12^{\rho}(|z|+3)^{\rho}, \quad z \in \mathbb{C}^{n},
$$

where $\xi$ is an arbitrary point of $\mathbb{S}$ with $F(\xi) \neq 0$. 
Proof. Let $z \in \mathbb{C}^{n}$. We choose a positive number $R$ with $|z|+1<R<|z|+2$. Since $B(\xi, 4 e R) \subset B(0,4 e(|z|+3))$, by (5.2) and the maximum of the modulus principle we have

$$
\ln \left|F\left(z^{\prime}\right) / F(\xi)\right| \leq C-\ln |F(\xi)|+D(4 e(|z|+3))^{\rho}, \quad z^{\prime} \in B(\xi, 4 e R) .
$$

Putting $M=C-\ln |F(\xi)|+D(4 e(|z|+3))^{\rho}, \eta=e^{-3}$, we choose $\varsigma \in \mathbb{S}$ in such a way that the complex line $\xi+\lambda \varsigma$ contains $z$. By Lemma 5.1, there exists $\tau \in(R, 2 R)$ such that

$$
\ln |F(\xi+\lambda \varsigma)|-\ln |f(\xi)| \geq-(4-\ln \eta) M=-7 M, \quad|\lambda|=\tau .
$$

We observe that $|\xi+\lambda \varsigma| \leq 2(|z|+3)$ whenever $|\lambda|=\tau$. Therefore, by the above and (5.2), we obtain

$$
\psi(\xi+\lambda \varsigma) \leq A+7 C-8 \ln |F(\xi)|+(7 D+B) 12^{\rho}(|z|+3)^{\rho},
$$

where $|\lambda|=\tau$. By the choice of $R$ and $\varsigma$, we have $z=\xi+\lambda \varsigma$ for some $\lambda$ with $|\lambda|<\tau$. The required estimate follows from the maximum principle and the preceding inequality.

Lemma 5.3. Let $\varphi(z)$ be an entire function of exponential type,

$$
\ln |\varphi(z)| \leq A+B|z|, \quad z \in \mathbb{C}^{n} .
$$

For every positive number $\delta$, there exists $a \geq 0$ such that for every $\varsigma \in \mathbb{S}$ we have

$$
\ln \left|\varphi\left(z_{k}\right)\right| \geq-a\left|z_{k}\right|, \quad k \geq 1,
$$

for some sequence $\left\{z_{k}\right\} \in \Phi_{\delta}(\varsigma)$.

Proof. We fix $\delta>0, \varsigma \in \mathbb{S}, k=1,2, \ldots$, and a point $\xi \in \mathbb{S}$ at which $\varphi(z)$ does not vanish. Put $R_{k}=\left(1+2^{-1} \delta\right)^{k}, \eta=\delta /(20(1+\delta))$, and $r_{k}=R_{k}(2+\delta) /(2(1+\delta))$. Then $\eta \in(0,1 / 9)$ and $r_{k} \in\left(0,(1-9 \eta) R_{k}\right)$. By (5.3) and Lemma 5.1, there exists $\tau_{k} \in\left(r_{k}, r_{k}+9 \eta R_{k}\right)$ such that

$$
\ln |\varphi(\xi+\lambda \varsigma)|-\ln |\varphi(\xi)| \geq-(4-\ln \eta) M, \quad|\lambda|=\tau_{k},
$$

where $M=A-\ln |\varphi(\xi)|+2 e B R_{k}$. Let $z_{k}=\xi+\tau_{k} \varsigma$. We show that $\left\{z_{k}\right\} \in \Phi_{2 \delta}(\varsigma)$. First, we observe that

$$
\frac{2+\delta}{2(1+\delta)}=1-\frac{\delta}{2(1+\delta)}>1-\frac{\delta}{2+\delta}=\frac{2}{2+\delta}=\frac{1}{1+\delta / 2} .
$$

By the definition of $R_{k}$ and $r_{k}$, there exists $k^{\prime}$ such that

$$
R_{k}>1+r_{k}+9 \eta R_{k}>1+\tau_{k} \geq\left|z_{k}\right| \geq \tau_{k}-1>r_{k}-1>R_{k-1}
$$

for $k \geq k^{\prime}$. Consequently, $\left|z_{k}\right| \rightarrow \infty$ as $k \rightarrow \infty$. It is easily seen that $z_{k} /\left|z_{k}\right| \rightarrow \varsigma, k \rightarrow \infty$. Formula (5.5) and the definition of $R_{k}$ and $r_{k}$ show that

$$
\frac{\left|z_{k+1}\right|}{\left|z_{k}\right|}<\frac{R_{k+1}}{r_{k}-1}=\frac{2 R_{k+1}(1+\delta)}{R_{k}(2+\delta)\left(1-1 / r_{k}\right)}=\frac{2 R_{k}(1+\delta)(1+\delta / 2)}{R_{k}(2+\delta)\left(1-1 / r_{k}\right)}=\frac{(1+\delta)}{\left(1-1 / r_{k}\right)} .
$$

Therefore, $\left\{z_{k}\right\}_{k=1}^{\infty} \in \Phi_{2 \delta}(\varsigma)$. Put $a=3 e B(4-\ln \eta)(1+\delta / 2)$ and $\alpha=5 \ln |\varphi(\xi)|-$ $(4-\ln \eta) A$. Then by (5.4) and (5.5), and since $B$ is nonnegative, there exists $k^{\prime \prime}$ such that

$$
\begin{aligned}
\ln \left|\varphi\left(z_{k}\right)\right| & \geq \alpha-2 e B(4-\ln \eta) R_{k}=\alpha-2 e B(1+\delta / 2)(4-\ln \eta) R_{k-1} \\
& \geq \alpha-2 e B(1+\delta / 2)(4-\ln \eta)\left|z_{k}\right| \geq-a\left|z_{k}\right|, \quad k \geq k^{\prime \prime} .
\end{aligned}
$$

Clearly, this finishes the proof of the lemma. 
Three statements that follow constitute the main content of this section. In them, special plurisubharmonic functions are constructed with prescribed asymptotic behavior.

Let $F$ be an entire function and $N$ its zero set. We denote by $\Delta_{F}$ the set of all limits of sequences of the form $\left\{z_{k} /\left|z_{k}\right|\right\}$, where $z_{k} \in N, k=1,2, \ldots$, and $\left|z_{k}\right| \rightarrow \infty$. It should be noted that $\Delta_{F}$ coincides with $\Delta(W)$ if $F=F_{W}$.

Proposition 5.1. Suppose that an entire function $F$ on $\mathbb{C}^{n}$, an entire function $f$ of exponential type, a constant $d_{1}>0$, and a compact convex set $L \subset \mathbb{C}^{n}$ are such that

$$
\ln |f(z)| \leq d_{1}+H_{L}(z), \quad z \in \mathbb{C}^{n}
$$

Suppose also that $f$ is divisible by $F$. Then for every $\varepsilon>0$ and every compact set $S_{0} \subset \mathbb{S} \backslash \Delta_{F}$ there exists a plurisubharmonic function $\psi$ on $\mathbb{C}^{n}$, a neighborhood $\Omega$ of $S_{0}$, and constants $a_{0}, T>0$ such that

1) $\ln |F(z)|+\psi(z) \leq d_{1}+H_{L}(z)+\varepsilon|z|, \quad z \in \mathbb{C}^{n}$

2) $\ln |F(z)|+\psi(z) \geq-a_{0}|z|$ for all $z \in \mathbb{C}^{n}$ with $z /|z| \in \Omega,|z| \geq T$.

Proof. First, we observe that if $S_{0}$ is the union of compact sets $S_{1}, \ldots, S_{p}$, and for each $S_{i}$, $i=1, \ldots, p$, we construct a plurisubharmonic function $\psi_{i}$ satisfying 1) and 2) for this $S_{i}$ in place of $S_{0}$, then we can take the maximum of these $\psi_{i}$ as the required function $\psi$ for $S_{0}$. Therefore, we can assume that $S_{0}$ is sufficiently small. It is convenient to assume that $S_{0}$ is included in some open half-space whose boundary hyperplane contains the origin. This hyperplane includes a complex hyperplane of the form $\{z: g(z)=\langle z, \lambda\rangle=0\}$, which, by its choice, does not intersect $S_{0}$. We may assume that $\lambda$ belongs to the sphere $\mathbb{S}$.

Now, we pass to the construction of $\psi$ itself. Put

$$
\psi^{\prime}(z)=\ln |f(z)|-\ln |F(z)| .
$$

By assumption, $\psi^{\prime}$ is plurisubharmonic on $\mathbb{C}^{n}$. Since $g(z)$ is analytic, for every positive $\delta$ the function

$$
\psi(z)=\left(\sigma_{n} \delta^{2 n}\right)^{-1} \int_{B(0, \delta)} \psi^{\prime}(z+g(z) w) d \sigma(w)
$$

is also plurisubharmonic. After the substitution $w^{\prime}=e^{i \varphi} w$, where $\varphi$ is an argument of $g(z)$, we obtain the formula

$$
\psi(z)=\left(\sigma_{n} \delta^{2 n}\right)^{-1} \int_{B(0, \delta)} \psi^{\prime}(z+|g(z)| w) d \sigma(w) .
$$

Fix $\varepsilon>0$. By Lemma 4.2, there exists $\delta \in(0,1)$ such that

$$
\sup _{w \in B(z, \delta|z|)} H_{L}(w) \leq H_{L}(z)+\varepsilon|z|, \quad z \in \mathbb{C}^{n} .
$$

Since $S_{0} \subset \mathbb{S} \backslash \Delta_{F}$ and $\Delta_{F}$ is a closed set, by reducing $\delta$ if necessary we may assume that $S_{0}^{3 \delta}$ (that is, the $3 \delta$-blowup of $S_{0}$ ) does not intersect $\Delta_{F}$. The balls $B(\varsigma, \delta / 3), \varsigma \in S_{0}$, cover $S_{0}$, so that we can extract a finite subcovering $B\left(\varsigma_{j}, \delta / 3\right), j=1, \ldots, j_{0}$. We define the required neighborhood $\Omega$ of $S_{0}$ to be the union of the balls $B\left(\varsigma_{j}, \delta / 3\right), j=1, \ldots, j_{0}$. We may also assume that $\alpha=\inf _{z \in \Omega}|g(z)|>0$. Applying Lemma 5.3, we find $a \geq 0$ and some sequences $\left\{z_{j, k}\right\} \in \Phi_{\delta / 4}\left(\varsigma_{j}\right), j=1, \ldots, j_{0}$, such that

$$
\ln \left|f\left(z_{j, k}\right)\right| \geq-a\left|z_{j, k}\right|, \quad k \geq 1, \quad j=1, \ldots, j_{0} .
$$

As has already been mentioned, we may assume that the sequences $\left\{\left|z_{j, k}\right|\right\}$ are monotone increasing and tend to $+\infty$. Using the definition of $\Phi_{\delta}(\varsigma)$, we choose an index $k_{0}$ with

$$
\left|z_{j, k} /\right| z_{j, k}\left|-\varsigma_{j}\right| \leq \delta / 3, \quad\left|z_{j, k+1}\right| /\left|z_{j, k}\right| \leq 1+\delta / 3, \quad k \geq k_{0}, \quad j=1, \ldots, j_{0} .
$$


Let $T \geq \max _{1 \leq j \leq j_{0}}\left|z_{j, k_{0}}\right|$. Since $S_{0}^{3 \delta}$ does not intersect $\Delta_{F}$, the definition of $\Delta_{F}$ shows that $T$ can be chosen in such a way that the ball $B(\varsigma, 8 \delta|\varsigma| / 3)$ does not intersect the zero set of $F$ if $|\varsigma| \geq T$ and $\varsigma /|\varsigma| \in S_{0}$. We fix $z \in \Omega \cap \mathbb{S}$ and $t \geq T$, and choose indices $j, k$ in such a way that $B\left(\varsigma_{j}, \delta / 3\right)$ includes the point $z$ and

$$
\left|z_{j, k-1}\right| \leq t \leq\left|z_{j, k}\right| \text {. }
$$

Then by (5.8) we obtain

$$
\left|z_{j, k}-t z\right| \leq\left|z_{j, k}-t z_{j, k} /\right| z_{j, k}||+\left|t \varsigma_{j}-t z_{j, k} /\right| z_{j, k}||+\left|t \varsigma_{j}-t z\right|<t \delta .
$$

Consequently, the ball $B(t z,|g(t z)| \delta)$ is included in $B\left(z_{j, k},(t+|g(t z)|) \delta\right)$. Moreover, by (5.9), (5.8), and the inequality $|g(t z)| \leq|t z||\lambda|=t$, we have

$$
B\left(z_{j, k},(t+|g(t z)|) \delta\right) \subseteq B\left(z_{j, k}, 2 t \delta\right) \subseteq B\left(z_{j, k}, 2\left|z_{j, k}\right| \delta\right) \subseteq B\left(\left|z_{j, k}\right| \varsigma, 7\left|z_{j, k}\right| \delta / 3\right) .
$$

Since $\left|z_{j, k}\right| \geq t \geq T$, it follows that the ball $\overline{B\left(z_{j, k},(t+|g(t z)|) \delta\right)}$ does not intersect the zero set of $F$, and therefore the function $\ln |F|$ is pluriharmonic in this ball. We use the mean value inequality (respectively, mean value theorem) for subharmonic (respectively, harmonic) functions to obtain

$$
\begin{aligned}
\ln \left|f\left(z_{j, k}\right)\right| \leq\left(\sigma_{n}(t+|g(t z)|)^{2 n} \delta^{2 n}\right)^{-1} \int_{B\left(z_{j, k},(t+|g(t z)|) \delta\right)} \ln |f(w)| d \sigma(w) \\
=\frac{\sigma_{n}|g(t z)|^{2 n} \delta^{2 n}}{\sigma_{n}(t+|g(t z)|)^{2 n} \delta^{2 n}}\left(\sigma_{n}|g(t z)|^{2 n} \delta^{2 n}\right)^{-1} \int_{B(t z,|g(t z)| \delta)}\left(\psi^{\prime}(w)+\ln |F(w)|\right) d \sigma(w) \\
\quad+\left(\sigma_{n}(t+|g(t z)|)^{2 n} \delta^{2 n}\right)^{-1} \int_{B\left(z_{j, k},(t+|g(t z)|) \delta\right) \backslash B(t z,|g(t z)| \delta)} \ln |f(w)| d \sigma(w) \\
=\frac{|g(t z)|^{2 n}}{(t+|g(t z)|)^{2 n}}(\psi(t z)+\ln |F(t z)|) \\
\quad+\left(\sigma_{n}(t+|g(t z)|)^{2 n} \delta^{2 n}\right)^{-1} \int_{B\left(z_{j, k},(t+|g(t z)|) \delta\right) \backslash B(t z,|g(t z)| \delta)} \ln |f(w)| d \sigma(w) .
\end{aligned}
$$

By (5.7) and the assumption about $\ln |f|$, this implies that

$$
-a\left|z_{j, k}\right| \leq \frac{|g(t z)|^{2 n}}{(t+|g(t z)|)^{2 n}}(\psi(t z)+\ln |F(t z)|)+d_{1}+\sup _{w \in B\left(z_{j, k},(t+|g(t z)|) \delta\right)} H_{L}(w) .
$$

Let $b=\sup _{|w|=1} H_{L}(w)$. The homogeneity of the support function and the inequalities $\left|z_{j, k}\right| \geq t \geq|g(t z)| \geq \alpha t,\left|z_{j, k}\right| \leq t(1+\delta)$, and $\delta<1$ show that

$$
\begin{aligned}
\psi\left(z^{\prime}\right)+\ln \left|F\left(z^{\prime}\right)\right| & \geq-\frac{(t+|g(t z)|)^{2 n}}{|g(t z)|^{2 n}}\left(a\left|z_{j, k}\right|+d_{1}+b\left(\left|z_{j, k}\right|+(|g(t z)|+t) \delta\right)\right) \\
& \geq-(2 / \alpha)^{2 n}\left(a+d_{1} /\left|z_{j, k}\right|+b(1+2 \delta)\right)\left|z_{j, k}\right| \\
& \geq-(2 / \alpha)^{2 n}\left(a+d_{1} / T+3 b\right)\left|z_{j, k}\right| \\
& \geq-(2 / \alpha)^{2 n}\left(a+d_{1} / T+3 b\right)\left|z^{\prime}\right|(1+\delta) \\
& \geq-a_{0}\left|z^{\prime}\right|, z^{\prime}=t z, \quad z \in \Omega \cap \mathbb{S}, \quad t \geq T,
\end{aligned}
$$

where $a_{0}=2(2 / \alpha)^{2 n}\left(a+d_{1} / T+3 b\right)$. This gives inequality 2$)$ in the statement.

On the other hand, using (5.6), the mean value inequality for subharmonic functions, the assumption about $\ln |f|$, the homogeneity of the support function, and the inequality 
$|g(z)| \leq|z|$, we obtain

$$
\begin{aligned}
\psi(z)+\ln |F(z)| & =\left(\sigma_{n} \delta^{2 n}\right)^{-1} \int_{B(0, \delta)} \psi^{\prime}(z+|g(z)| w) d \sigma(w)+\ln |F(z)| \\
& \leq\left(\sigma_{n} \delta^{2 n}\right)^{-1} \int_{B(0, \delta)} \psi^{\prime}(z+|g(z)| w)+\ln |F(z+|g(z)| w)| d \sigma(w) \\
& \leq d_{1}+\sup _{w \in B(z,|g(z)| \delta)} H_{L}(w) \leq d_{1}+\sup _{w \in B(z,|z| \delta)} H_{L}(w) \\
& \leq d_{1}+H_{L}(z)+\varepsilon|z|, \quad z \in \mathbb{C}^{n} .
\end{aligned}
$$

This completes the proof of the proposition.

The next statement is a modification of the preceding one. Under the assumption of supplementary lower estimates on $\ln |f(z)|$, an inequality sharper than 2) in Proposition 5.1 is claimed in it.

Proposition 5.2. Suppose $F$ is an entire function and $\psi_{0}$ is a plurisubharmonic function on $\mathbb{C}^{n}$. Suppose also that

$$
\psi_{0}(z)+\ln |F(z)| \leq d+H_{L}(z), \quad z \in \mathbb{C}^{n},
$$

for some constant $d$ and some compact convex set $L \subset \mathbb{C}^{n}$.

Next, let $\varsigma \in \mathbb{S} \backslash \Delta_{F}$, and let

$$
\psi_{0}\left(z_{k}\right)+\ln \left|F\left(z_{k}\right)\right| \geq l\left(z_{k}\right), \quad k \geq 1
$$

for some sequence $\left\{z_{k}\right\} \in \Phi_{0}(\varsigma)$, where $l(z)$ is a continuous function that is positive homogeneous of degree 1 . Then for every $\varepsilon>0$ there exists a function $\psi$ plurisubharmonic in $\mathbb{C}^{n}$ and some constants $d_{1}, \delta, T>0$ such that

1) $\ln |F(z)|+\psi(z) \leq d_{1}+H_{L}(z)+\varepsilon|z|, \quad z \in \mathbb{C}^{n}$;

2) for all $z \in B(\varsigma, \delta) \cap \mathbb{S}$ and $t \geq T$ we have

$$
\ln |F(t z)|+\psi(t z) \geq H_{L}(t z)+8^{2 n}\left(l(t z)-3 \varepsilon t-H_{L}(t z)\right) .
$$

Proof. Take $\lambda \in \mathbb{S}$ such that the complex plane $g(z)=\langle z, \lambda\rangle=0$ does not intersect the ball $B(\varsigma, 1)$. Then

$$
\inf _{z \in B(\varsigma, 1 / 2)}|g(z)| \geq 1 / 2 .
$$

Fixing $\varepsilon>0$, by Lemma 4.2 we find $\delta \in(0,1 / 2)$ with

$$
\begin{gathered}
\sup _{w \in B(z, 7 \delta|z|)} H_{L}(w) \leq H_{L}(z)+\varepsilon|z|, \quad z \in \mathbb{C}^{n}, \\
\sup _{w \in B(z, 4 \delta|z|)} l(w) \leq l(z)+\varepsilon|z|, \quad z \in \mathbb{C}^{n} .
\end{gathered}
$$

We may assume that $B(\varsigma, 6 \delta) \cap \Delta_{F}=\varnothing$. The definition of the sets $\Delta_{F}$ and $\Phi_{0}(\varsigma)$ shows that there exists $T>\left|z_{1}\right|$ such that the ball $\overline{B(t \varsigma, 5 t \delta)}$ does not intersect the zero set of $F$ for $t \geq T$ and $\left|z_{k}\right| /\left|z_{k-1}\right|<1+\delta$ if $\left|z_{k}\right| \geq T$. We put

$$
\psi(z)=\left(\sigma_{n} \delta^{2 n}\right)^{-1} \int_{B(0, \delta)} \psi_{0}(z+|g(z)| w) d \sigma(w) .
$$

Much as in Proposition 5.1, we prove that $\psi(z)$ is plurisubharmonic and satisfies 1). Now we show that 2) is also true.

Fixing $z \in B(\varsigma, \delta) \cap \mathbb{S}$ and $t \geq T$, we choose $k$ with $\left|z_{k-1}\right| \leq t \leq\left|z_{k}\right|$. As in Proposition 5.1, we verify the inequality $\left|z_{k}-t z\right|<3 t \delta$ and the fact that $\ln |F|$ is pluriharmonic 
in the ball $\overline{B\left(z_{k},(3 t+|g(t z)|) \delta\right)}$. Increasing $T>0$ if necessary, we may assume that $\varepsilon t \geq d$ if $t \geq T$. Since $|g(t z)| \leq t \leq\left|z_{k}\right|$, formulas (5.10) and (5.13) imply that

$$
\begin{aligned}
q(w) & =\psi_{0}(w)+\ln |F(w)|-H_{L}(t z)-2 \varepsilon t \leq 0, \\
w & \in B\left(z_{k},(3 t+|g(t z)|) \delta\right) \subset B(t z, 7 t \delta) .
\end{aligned}
$$

Therefore, using (5.11), the mean value inequality for subharmonic functions, and the embedding $B(t z,|g(t z)| \delta) \subset B\left(z_{k},(3 t+|g(t z)|) \delta\right)$, we see that

$$
\begin{aligned}
l\left(z_{k}\right)-H_{L}(t z)-2 \varepsilon t & \leq q\left(z_{k}\right) \leq\left(\sigma_{n}((3 t+|g(t z)|) \delta)^{2 n}\right)^{-1} \int_{B\left(z_{k},(3 t+|g(t z)|) \delta\right)} q(w) d \sigma(w) \\
& \leq \frac{\sigma_{n}(|g(t z)| \delta)^{2 n}}{\sigma_{n}((3 t+|g(t z)|) \delta)^{2 n}}\left(\sigma_{n}(|g(t z)| \delta)^{2 n}\right)^{-1} \int_{B(t z,|g(t z)| \delta)} q(w) d \sigma(w) .
\end{aligned}
$$

By (5.14) and the mean value theorem for harmonic functions, we find

$$
\begin{aligned}
l(t z)-3 \varepsilon t-H_{L}(t z) & \leq l\left(z_{k}\right)-H_{L}(t z)-2 \varepsilon t \\
& \leq \frac{|g(t z)|^{2 n}}{(3 t+|g(t z)|)^{2 n}}\left(\psi(t z)+\ln |F(t z)|-H_{L}(t z)-2 \varepsilon t\right) .
\end{aligned}
$$

Since $|g(t z)| \leq t$ and also $|g(t z)| \geq t / 2$ by (5.12), finally we obtain

$$
\begin{aligned}
\psi(t z)+\ln |F(t z)| & \geq H_{L}(t z)+2 \varepsilon t+8^{2 n}\left(l(t z)-3 \varepsilon t-H_{L}(t z)\right) \\
& \geq H_{L}(t z)+8^{2 n}\left(l(t z)-3 \varepsilon t-H_{L}(t z)\right) .
\end{aligned}
$$

Proposition 5.3. Let $F$ be an entire function, $D$ a convex domain, and $S_{0}$ a compact subset of $\mathbb{S}$ that has no points in common with $\Delta_{F} \cup \Theta_{D}$. Suppose that for every $\varsigma \in S_{0}$ and every index $m$ there exists a plurisubharmonic function $\psi_{\varsigma, m}$ such that

$$
\begin{aligned}
& h_{u_{\varsigma, m}}(z)<H_{D}(z), \quad z \in \mathbb{C}^{n} \backslash\{0\}, \\
& \underline{h}_{u_{\varsigma, m}}(\varsigma) \geq H_{K_{m}}(\varsigma),
\end{aligned}
$$

where $u_{\varsigma, m}=\ln |F|+\psi_{\varsigma, m}$. Then for every index $m$ there exists a function $\psi$ plurisubharmonic in $\mathbb{C}^{n}$, a neighborhood $\Omega$ of $S_{0}$, and a constant $T>0$ such that

1) $h_{u}(z)<H_{D}(z), z \in \mathbb{C}^{n} \backslash\{0\}$, where $u=\ln |F|+\psi$;

2) $u(z) \geq H_{K_{m}}(z)$ for all $z \in \mathbb{C}^{n}$ satisfying the conditions $z /|z| \in \Omega,|z| \geq T$.

Proof. We fix an index $m$. Since the compact set $K_{m}$ is included in the interior of $K_{m+1}$, for some $\alpha>0$ we have

$$
H_{K_{m}}(z)+\alpha|z| \leq H_{K_{m+1}}(z), \quad z \in \mathbb{C}^{n} .
$$

Let $\varsigma \in S_{0}$. By assumption, $H_{D}(\varsigma)<\infty$. Since $D=\bigcup K_{j}$, there exists an index $m(\varsigma) \geq$ $m+1$ with

$$
H_{K_{m(\varsigma)}}(\varsigma)+8^{-(2 n+1)} \alpha \geq H_{D}(\varsigma) .
$$

Also, by assumption, there exists a plurisubharmonic function $\psi_{\varsigma, m(\varsigma)}$ such that (5.15) and (5.16) are true for $m_{\varsigma}, m_{(\varsigma)}$. By (5.15), there is $d(\varsigma)>0$ and a compact set $L(\varsigma) \subset D$ satisfying

$$
u_{\varsigma, m(\varsigma)}(z) \leq d(\varsigma)+H_{L(\varsigma)}(z), \quad z \in \mathbb{C}^{n} .
$$

We choose a positive number $\varepsilon(\varsigma) \leq 8^{-(2 n+1)} \alpha$ in such a way that

$$
H_{L(\varsigma)}(z)+2 \varepsilon(\varsigma)|z| \leq H_{D}(z), \quad z \in \mathbb{C}^{n} .
$$

By (5.16) and Lemma 2.7 in [30, for some sequence $\left\{z_{k}\right\} \in \Phi_{0}(\varsigma)$ we have

$$
\liminf _{k \rightarrow \infty} u_{\varsigma, m(\varsigma)}\left(z_{k}\right) /\left|z_{k}\right| \geq H_{K_{m}}(\varsigma) \text {. }
$$


Since the support function of a compact set is continuous and positive homogeneous, we may assume that

$$
u_{\varsigma, m(\varsigma)}\left(z_{k}\right) \geq H_{K_{m}}\left(z_{k}\right)-\varepsilon(\varsigma)\left|z_{k}\right|, \quad k=1,2, \ldots
$$

Therefore, the assumptions of Proposition 5.2 are satisfied. By that proposition, there exist functions $\psi_{\varsigma}$ plurisubharmonic in $\mathbb{C}^{n}$ and constants $d_{1}(\varsigma), \delta(\varsigma), T(\varsigma)>0$ such that

$$
u_{\varsigma}(z) \leq d_{1}(\varsigma)+H_{L(\varsigma)}(z)+\varepsilon(\varsigma)|z|, \quad z \in \mathbb{C}^{n},
$$

where $u_{\varsigma}=\ln |F|+\psi_{\varsigma}$, and

$$
u_{\varsigma}(t z) \geq H_{L(\varsigma)}(t z)+8^{2 n}\left(H_{K_{m(\varsigma)}}(t z)-4 \varepsilon(\varsigma) t-H_{L(\varsigma)}(t z)\right),
$$

for all $z \in B(\varsigma, \delta(\varsigma)) \cap \mathbb{S}, t \geq T(\varsigma)$. Formula (5.18) implies that

$$
H_{K_{m(\varsigma)}}(\varsigma)-H_{L(\varsigma)}(\varsigma) \geq H_{K_{m(\varsigma)}}(\varsigma)-H_{D}(\varsigma) \geq-8^{-(2 n+1)} \alpha .
$$

Therefore, taking the continuity of the support function into account and reducing $\delta(\varsigma)>0$ if necessary, we may assume that

$$
H_{K_{m(\varsigma)}}(t z)-H_{L(\varsigma)}(t z) \geq 8^{-(2 n+1)} \alpha t, \quad z \in B(\varsigma, \delta(\varsigma)) \cap \mathbb{S}, \quad t \geq 0 .
$$

Then by the above and the choice of $\varepsilon(\varsigma)$, we obtain

$$
u_{\varsigma}(t z) \geq H_{L(\varsigma)}(t z)-6 \cdot 8^{-1} \alpha t, \quad z \in B(\varsigma, \delta(\varsigma)) \cap \mathbb{S}, \quad t \geq T(\varsigma) .
$$

On the other hand, (5.16) and (5.19) imply that

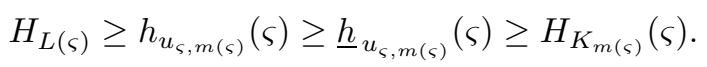

Thus, using (5.17) and the inclusion $K_{m+1} \subset K_{m(\varsigma)}$ (which is implied by $m(\varsigma) \geq m+1$ ), we finally obtain

$$
\begin{aligned}
u_{\varsigma}(t z) & \geq H_{L(\varsigma)}(t z)-6 \cdot 8^{-1} \alpha t \geq H_{K_{m(\varsigma)}}(t z)-\alpha t / 4-3 \alpha t / 4 \\
& \geq H_{K_{m(\varsigma)}}(t z)-\alpha t \geq H_{K_{m}}(t z), \quad z \in B(\varsigma, \delta(\varsigma)) \cap \mathbb{S}, \quad t \geq T(\varsigma) .
\end{aligned}
$$

Now, we pass to the construction of $\psi$. We extract a finite subcovering $B\left(\varsigma_{j}, \delta\left(\varsigma_{j}\right)\right)$, $j=1, \ldots, p$, from the covering $B(\varsigma, \delta(\varsigma)), \varsigma \in S_{0}$, and put

$$
\Omega=\bigcup_{j=1}^{p} B\left(\varsigma_{j}, \delta\left(\varsigma_{j}\right)\right), \quad T=\max _{1 \leq j \leq p} T\left(\varsigma_{j}\right), \quad \psi(z)=\max _{1 \leq j \leq p} \psi_{\varsigma_{j}}(z) .
$$

By (5.20) and (5.21), $u(z)$ satisfies statement 1$)$ in the proposition; by (5.22), it also satisfies 2), and the proof is complete.

At the end of the section, we present yet another construction of a special plurisubharmonic function.

Lemma 5.4. Suppose that plurisubharmonic functions $g$ and $\psi_{0}$ on $\mathbb{C}^{n}$, a constant $d_{0}>0$, and a compact convex set $L \subset \mathbb{C}^{n}$ are such that

$$
\psi_{0}(z)+g(z) \leq d_{0}+H_{L}(z), \quad z \in \mathbb{C}^{n} .
$$

Then there exists a continuous plurisubharmonic function $\psi \geq \psi_{0}$ on $\mathbb{C}^{n}$ such that

$$
h_{\psi+g}(z) \leq H_{L}(z), \quad z \in \mathbb{C}^{n} .
$$

Proof. Put

$$
\psi(z)=\sigma_{n}^{-1} \int_{B(0,1)} \psi_{0}(z+w) d \sigma(w)
$$


The function $\psi(z)$ is plurisubharmonic; by the mean value inequality for subharmonic functions we have $\psi(z) \geq \psi_{0}(z), z \in \mathbb{C}^{n}$, and

$$
\begin{aligned}
\psi(z)+g(z) & \leq \sigma_{n}^{-1} \int_{B(0,1)} \psi_{0}(z+w) d \sigma(w)+\sigma_{n}^{-1} \int_{B(0,1)} g(z+w) d \sigma(w) \\
& \leq \sup _{w \in B(z, 1)}\left(\psi_{0}(w)+g(w)\right) \leq d_{0}+\sup _{w \in B(z, 1)} H_{L}(w), \quad z \in \mathbb{C}^{n} .
\end{aligned}
$$

Here the last inequality follows from (5.23). By Lemma 4.2, for every $\beta>0$ there exists $\delta>0$ such that

$$
\sup _{w \in \overline{B(z, \delta|z|)}} H_{L}(w) \leq H_{L}(z)+\beta|z|, \quad z \in \mathbb{C}^{n} .
$$

We choose $T>0$ with $T \delta>1$. Then (5.24) implies that

$$
\psi(z)+g(z) \leq d_{0}+H_{L}(z)+\beta|z|, \quad|z|>t .
$$

Since the support function of a compact set is continuous, the definition of the upper indicator yields

$$
h_{\psi+g}(z) \leq H_{L}(z)+\beta|z|, \quad z \in \mathbb{C}^{n} .
$$

This implies the required estimate because $\beta>0$ is arbitrary. It remains to prove that $\psi(z)$ is continuous. Fix $z_{0} \in \mathbb{C}^{n}$. Since the function $\psi_{0}(z)$ is plurisubharmonic, the quantity

$$
c_{1}=\sigma_{n}^{-1} \int_{B(0,2)} \psi_{0}\left(z_{0}+w\right) d \sigma(w)
$$

is finite. We choose $c_{2}$ with $\psi_{0}(w) \leq c_{2}$ for all $w \in B\left(z_{0}, 4\right)$. Let $z \in B\left(z_{0}, 1\right)$. Then

$$
\begin{aligned}
c_{1} & =\sigma_{n}^{-1} \int_{B(0,2)} \psi_{0}\left(w+z_{0}\right) d \sigma(w) \\
& =\sigma_{n}^{-1}\left(\int_{B(z, 1)} \psi_{0}(w) d \sigma(w)+\int_{B(0,2) \backslash B(z, 1)} \psi_{0}(w) d \sigma(w)\right) \\
& \leq \sigma_{n}^{-1} \int_{B(z, 1)} \psi_{0}(w) d \sigma(w)+c_{2}\left(2^{2 n}-1\right) .
\end{aligned}
$$

Consequently,

$$
\psi(z)=\sigma_{n}^{-1} \int_{B(z, 1)} \psi_{0}(w) d \sigma(w) \geq c_{1}-c_{2}\left(2^{2 n}-1\right)=c_{3}, \quad z \in B\left(z_{0}, 1\right) .
$$

Together with (5.25), this implies that

$$
\begin{aligned}
\psi\left(z_{1}\right) & =\sigma_{n}^{-1} \int_{B\left(z_{1}, 1\right)} \psi_{0}(w) d \sigma(w) \leq\left((1+\rho)^{2 n} \sigma_{n}\right)^{-1} \int_{B\left(z_{1}, 1+\rho\right)} \psi_{0}(w) d \sigma(w) \\
& \leq\left((1+\rho)^{2 n} \sigma_{n}\right)^{-1} \int_{B\left(z_{2}, 1\right)} \psi_{0}(w) d \sigma(w)+\frac{(1+\rho)^{2 n}-1}{(1+\rho)^{2 n}} c_{2} \\
& \leq(1+\rho)^{-2 n} \psi\left(z_{2}\right)+\frac{(1+\rho)^{2 n}-1}{(1+\rho)^{2 n}} c_{2} .
\end{aligned}
$$

A similar estimate holds true if we interchange $z_{1}$ and $z_{2}$. Thus, we arrive at

$$
\begin{aligned}
\psi\left(z_{1}\right)-\psi\left(z_{2}\right) & \leq\left(\frac{1}{(1+\rho)^{2 n}}-1\right) \psi\left(z_{2}\right)+\frac{(1+\rho)^{2 n}-1}{(1+\rho)^{2 n}} c_{2} \\
& \leq\left(\frac{1}{(1+\rho)^{2 n}}-1\right) c_{3}+\frac{(1+\rho)^{2 n}-1}{(1+\rho)^{2 n}} c_{2} \leq c_{4} \rho .
\end{aligned}
$$




\section{$\S 6$. Geometry of Convex domains}

In this section we gather certain results about convex domains; they will be needed in the sequel.

Lemma 1. Let $D, G$ be convex domains in $\mathbb{C}^{n}$ with $D \subset G$. For every $\xi \in \mathbb{S} \backslash \Xi$ $(\Xi=\Xi(D, G))$ and $\gamma>0$, there exists $y \in G$ such that

1) $\operatorname{Re}\langle y, \xi\rangle=H_{D}(\xi)$;

2) $\operatorname{Re}\langle y, z\rangle \leq H_{D}(z)+\gamma|z|, z \in \mathbb{C}^{n}$.

Proof. First, we observe that if the claim holds true for some domains $D$ and $G$, then it holds true for an arbitrary shift of $D$ and $G$, i.e., for the domains $x+D$ and $x+G$, where $x$ is an arbitrary vector. Indeed, if $y \in G$ is the point as in the claim that corresponds to $D, G$, and a direction $\xi$, then $y+x$ will play the same role for $D+x$ and $G+x$, because this transformation results in adding $\operatorname{Re}\langle x, \xi\rangle$ to the two sides of 1 ) and $\operatorname{Re}\langle x, z\rangle$ to the two sides of 2 ). Thus, we may assume that $D$ contains the origin, in which case

$$
H_{D}(\varsigma)>0, \quad \varsigma \neq 0 .
$$

Fix $\xi \in \mathbb{S} \backslash \Xi$. We observe that $\xi \notin \Theta_{D}$, because $H_{D}(\xi)<H_{G}(\xi)$. Using the definition of the support function, we find $w \in G$ and a positive number $\alpha$ such that

$$
H_{D}(\xi)+\alpha<\operatorname{Re}\langle w, \xi\rangle .
$$

Next, again by the definition of the support function, we find a sequence $\left\{x_{k}\right\} \subset D$ with

$$
0>\operatorname{Re}\left\langle x_{k}, \xi\right\rangle-H_{D}(\xi)=-\varepsilon_{k} \rightarrow 0, \quad k \rightarrow \infty .
$$

Since $D \subset G$ and $G$ is convex, for every $k=1,2, \ldots$, the interval $\left[x_{k}, w\right]$ is included in $G$. Furthermore, $(6.2)$ and (6.3) show that for every $k=1,2, \ldots$, the interval $\left[x_{k}, w\right]$ contains a point $y_{k}$ with

$$
\operatorname{Re}\left\langle y_{k}, \xi\right\rangle=H_{D}(\xi)
$$

We fix $\gamma>0$ and prove that some of the $y_{k}$ can be taken for the required point $y$. By (6.4), for this it suffices to verify 2) with some $k$. For $k=1,2, \ldots$, we choose $\tau_{k} \in[0,1]$ such that $y_{k}=\tau_{k} x_{k}+\left(1-\tau_{k}\right) w$. Applying (6.4), we obtain

$$
\operatorname{Re}\left\langle y_{k}, \xi\right\rangle=\tau_{k} \operatorname{Re}\left\langle x_{k}, \xi\right\rangle+\left(1-\tau_{k}\right) \operatorname{Re}\langle w, \xi\rangle=H_{D}(\xi) .
$$

Together with (6.2) and (6.3), this implies the inequality $\tau_{k}>\alpha /\left(\alpha+\varepsilon_{k}\right), k=1,2, \ldots$

We choose $k$ in such a way that $\varepsilon_{k}|w| /\left(\alpha+\varepsilon_{k}\right)<\gamma$. Let $\varsigma \in \mathbb{S}$. Using (6.2) and the relations $x_{k} \in D, \tau_{k} \in[0,1]$, we obtain

$$
\begin{aligned}
\operatorname{Re}\left\langle y_{k}, \varsigma\right\rangle & =\tau_{k} \operatorname{Re}\left\langle x_{k}, \varsigma\right\rangle+\left(1-\tau_{k}\right) \operatorname{Re}\langle w, \varsigma\rangle \\
& <H_{D}(\varsigma)+|w| \varepsilon_{k} /\left(\alpha+\varepsilon_{k}\right) \leq H_{D}(\varsigma)+\gamma
\end{aligned}
$$

Since the support function is positive homogeneous, this yields 2) with $y=y_{k}$.

Lemma 6.2. Let $D, G$ be convex domains in $\mathbb{C}^{n}$ with $D \subset G$, and let $V^{\prime}$ be a neighborhood of the set $\mathbb{S} \cap \Xi \backslash$ int $\Theta_{D}$. Then the set $S_{0}=\left(\partial V^{\prime} \backslash \Theta_{D}\right) \cap \mathbb{S}$ is compact.

Proof. It suffices to show that $S_{0}$ is closed. Let a sequence $\left\{\xi_{m}\right\} \subset S_{0}$ converge to $\xi_{0}$. Then $\xi_{0} \in \partial V^{\prime} \cap \mathbb{S}$. If $\xi_{0} \notin \Theta_{D}$, then $\xi_{0} \in S_{0}$ and the proof is finished. Suppose that $\xi_{0} \in \Theta_{D}$. Then either $\xi_{0} \in \operatorname{int} \Theta_{D}$ or $\xi_{0} \in \partial \Theta_{D}$. In the former case all $\left\{\xi_{m}\right\}$ starting with some $m$ belong to int $\Theta_{D} \subset \Theta_{D}$, which contradicts the definition of $S_{0}$. In the latter case $\xi_{0} \in \partial \Theta_{D} \cap \Theta_{D}$. Since $\Theta_{D} \subset \Xi$, we also have $\xi_{0} \in\left(\Xi \backslash\right.$ int $\left.\Theta_{D}\right) \cap \mathbb{S}$, which says that $\xi_{0} \in V^{\prime}$. But this is impossible because $\xi_{0} \in \partial V^{\prime}$. This contradiction completes the proof. 
Lemma 6.3. Let $\Theta$ be a cone in $\mathbb{C}^{n}$ with vertex at the origin, and let $S_{0} \subset \mathbb{S}$ be a compact subset of the interior of $\Theta$. Suppose that the set $\mathbb{C}^{n} \backslash \Theta$ is convex. Then for every $A>0$ there exists a neighborhood $\Omega_{0}$ of $S_{0}$ and a convex function $\psi_{0}$ positive homogeneous of degree 1 with the following properties:

1) $\psi_{0}(z)=0, z \notin \Theta$

2) $\psi_{0}(z) \geq A|z|$ if $z /|z| \in \Omega_{0}$;

3) $\psi_{0}(z) \geq 0, z \in \mathbb{C}^{n}$.

Proof. Fix $A>0$ and take $\varsigma \in S_{0}$. Since $S \subset \operatorname{int} \Theta$, for some $\tau>0$ the ball $B(\varsigma, \tau)$ is included in $\Theta$. The set $\mathbb{C}^{n} \backslash \Theta$ is convex by assumption. By the separation theorem, there exists a (real) hyperplane $\Gamma$ that separates $B(\varsigma, \tau)$ and $\mathbb{C}^{n} \backslash \Theta$. Since the latter set is a cone with vertex at the origin, this hyperplane can be chosen so that it contains the origin. Let

$$
\Gamma=\{z: \operatorname{Re}\langle z, \lambda(\varsigma)\rangle=0\} .
$$

Changing the sign of $\lambda(\varsigma)$ if necessary, we may assume that

$$
\operatorname{Re}\langle z, \lambda(\varsigma)\rangle \leq 0, \quad z \notin \Theta,
$$

and $\operatorname{Re}\langle z, \lambda(\varsigma)\rangle \geq 0$ for all $z \in B(\varsigma, \tau)$. Then $\operatorname{Re}\langle z, \lambda(\varsigma)\rangle>0$. We choose a positive number $c(\varsigma)$ such that for the function $\psi_{\varsigma}(z)=c(\varsigma) \operatorname{Re}\langle z, \lambda(\varsigma)\rangle$ we have the estimate $\psi_{\varsigma}(\varsigma)>A=A|\varsigma|$. By continuity, this estimate extends to some neighborhood of $\varsigma$ :

$$
\psi_{\varsigma}(z) \geq A|z|, \quad z \in B(\varsigma, \delta(\varsigma)) .
$$

We extract a finite subcovering $B\left(\varsigma_{j}, \delta\left(\varsigma_{j}\right)\right), j=1, \ldots, p$, from the covering of $S_{0}$ by the balls $B(\varsigma, \delta(\varsigma)), \varsigma \in S_{0}$, and put

$$
\Omega_{0}=\bigcup_{j=1}^{p} B\left(\varsigma_{j}, \delta\left(\varsigma_{j}\right)\right), \quad \psi(z)=\max _{1 \leq j \leq p} \psi_{\varsigma_{j}}(z), \quad \psi_{0}(z)=\max \{\psi(z), 0\} .
$$

Clearly, the function $\psi_{0}(z)$ is convex and positive homogeneous of degree 1 . The definition of $\psi_{0}(z)$ readily implies statement 3 ) of the lemma. Recalling also (6.5), we verify statement 1$)$. Statement 2 ) follows by (6.6) and the fact that $\psi_{0}(z)$ is positive homogeneous.

Lemma 6.4. Let $D, G$ be convex domains with $D \subset G$. Then $G \subset G(D)$ and $\Xi(D, G)=$ $\Xi(D, G(D))$.

Proof. Let $z \in G$. Then $\operatorname{Re}\langle z, \xi\rangle<H_{G}(\xi), \xi \in \mathbb{S}$. Consequently, by the definition of $\Xi(D, G)$ we obtain

$$
\operatorname{Re}\langle z, \xi\rangle<H_{G}(\xi)=H_{D}(\xi), \quad \xi \in \Xi(D, G) .
$$

This shows that $z \in G(D)$. Thus, $G \subset G(D)$.

The last inclusion implies that

$$
H_{D}(\xi)<H_{G}(\xi) \leq H_{G(D)}(\xi), \quad \xi \in \mathbb{S} \backslash \Xi(D, G) .
$$

Therefore, $\Xi(D, G(D)) \subset \Xi(D, G)$. Now let $\xi \in \Xi(D, G)$. The definition of $G(D)$ shows that $H_{G(D)}(\xi) \leq H_{D}(\xi)$. On the other hand, the inclusion $D \subset G(D)$ implies the reverse inequality $H_{D}(\xi) \leq H_{G(D)}(\xi)$. Thus, $H_{G(D)}(\xi)=H_{D}(\xi)$, i.e., $\xi \in \Xi(D, G(D))$. The lemma follows. 


\section{§7. CONDITIONS NECESSARY FOR CONTINUABILITY}

Our main goal in this section is to exhibit some conditions necessary for the analytic continuability of all functions in a principal invariant subspace $W \subset H(D)$ to a convex domain $G$ that includes $D$ and is included in $D(W)$.

Theorem 7.1. Let $D$ be a convex domain in $\mathbb{C}^{n}, W$ a principal invariant subspace in $H(D)$ that admits spectral synthesis, and $G$ a convex subdomain of $D(W)$ containing $D$. Suppose that every function in $W$ admits analytic continuation to $G$ and, on $G$, can be approximated by linear combinations of elements of $E(W)$. Then for every $\xi \in \mathbb{S} \backslash \Xi$ and every index $m$ there exists a plurisubharmonic function $\psi$, an index $p$, and numbers $d, R, \delta>0$ with the following properties:

1) $\psi(z)+\ln \left|F_{W}(z)\right| \leq d+H_{K_{p}}(z), z \in \mathbb{C}^{n}$;

2) $\psi(z)+\ln \left|F_{W}(z)\right| \geq H_{K_{m}}(z)$ for all $z$ with $z /|z| \in B(\xi, \delta),|z|>R$.

Remark. The necessary conditions in Theorem 7.1 are similar to the sufficient conditions in Theorem 4.1. In the two theorems, the existence of plurisubharmonic functions with appropriate upper and lower estimates is required. The upper estimates are the same, but the lower estimates differ. In Theorem 4.1, lower estimates must be fulfilled in a neighborhood of a surface that "encompasses" the zero set $N(W)$. In Theorem 7.1, such estimates hold true only "locally" in a cone generated by a small neighborhood of a point $\xi \in \mathbb{S} \backslash \Xi$. It should also be noted that if $\Xi$ is not closed, a part of the surface occurring in Theorem 4.1 may lie inside $\Xi$; this means that, in distinction to Theorem 7.1, lower estimates must also be fulfilled on a certain subset of $\Xi$.

Proof. We fix an index $m$ and a point $\xi \in \mathbb{S} \backslash \Xi$. Since $K_{m}$ is a compact subset of $D$, there exists $\varepsilon>0$ with

$$
H_{K_{m}}(z)+\varepsilon|z| \leq H_{D}(z), \quad z \in \mathbb{C}^{n} .
$$

The subspace $W$ is nontrivial; therefore $I_{W}$ is also nontrivial. Let $\varphi_{1}$ be a nonzero element of $I_{W} \subset P_{D}$. The definition of $P_{D}$ implies the existence of a compact convex set $L_{1} \subset D$ and constants $d_{1}, \alpha>0$ such that

$$
\ln \left|\varphi_{1}(z)\right| \leq d_{1}+H_{L_{1}}(z) \leq d_{1}+H_{D}(z)-3 \alpha|z|, \quad z \in \mathbb{C}^{n} .
$$

Since $\varphi_{1}$ is divisible by $F_{W}$ (because $W$ admits spectral synthesis, see the remark to Lemma 3.1), by Proposition 5.1 there exists a function $\psi_{1}$ plurisubharmonic in $\mathbb{C}^{n}$, a number $\delta^{\prime} \in(0,1)$, and two constants $a_{0}, T>0$ such that

$$
\begin{aligned}
& \ln \left|F_{W}(z)\right|+\psi_{1}(z) \leq d_{1}+H_{L_{1}}(z)+\alpha|z|, \quad z \in \mathbb{C}^{n} \\
& \ln \left|F_{W}(z)\right|+\psi_{1}(z) \geq-a_{0}|z|, \quad z /|z| \in B\left(\xi, \delta^{\prime}\right), \quad|z| \geq T .
\end{aligned}
$$

Using (7.1), we choose $\tau \in(0,1)$ to satisfy the conditions

$$
(1-\tau) H_{D}(\xi)>H_{K_{m}}(\xi)+3 \varepsilon / 4 \text {. }
$$

Let $\gamma>0$ be such that

$$
(1-\tau) \gamma-\tau \alpha<0 .
$$

Lemma 6.1 implies the existence of $y \in G$ for which statements 1) and 2) of that lemma are fulfilled. Then the function $h(z)=\exp \langle y, z\rangle$ belongs to $P_{G}$. By Lemma 3.2, there exist functions $\varphi_{2} \in I_{W(G)}$ and $g \in P_{D}$ with $h=\varphi_{2}+g$. Since $g \in P_{D}$, there exists a compact convex set $L \subset D$ and a constant $d^{\prime}>0$ with

$$
\ln |g(z)| \leq d^{\prime}+H_{L}(z), \quad z \in \mathbb{C}^{n} .
$$


Let $L_{2}$ be the convex hull of $L \cup\{y\}$, and let $d_{2}=1+d^{\prime}$. Taking the inclusion $L \subset D$ and statement 2) of Lemma 6.1 into account, we obtain

$$
\begin{aligned}
\ln \left|\varphi_{2}(z)\right| & =\ln |h(z)-g(z)| \leq \ln (|h(z)|+|g(z)|) \\
& \leq \ln 2+\ln (\max [|h(z)|,|g(z)|]) \leq 1+d^{\prime}+\max \left[\operatorname{Re}\langle y, z\rangle, H_{L}(z)\right] \\
& =d_{2}+H_{L_{2}}(z) \leq d_{2}+H_{D}(z)+\gamma|z|, \quad z \in \mathbb{C}^{n} .
\end{aligned}
$$

Put $\psi_{2}(z)=\ln \left|\varphi_{2}(z)\right|-\ln \left|F_{W}(z)\right|$. Since $W(G)$ admits spectral synthesis, we deduce (see the remark to Lemma 3.1) that the function $\varphi_{2} \in I_{W(G)}$ is divisible by $F_{W}$. Consequently, $\psi_{2}(z)$ is a plurisubharmonic function. Let $\psi(z)=\tau \psi_{1}(z)+(1-\tau) \psi_{2}(z)$. By (7.3), (7.8), and (7.2) we have

$$
\begin{aligned}
\ln \left|F_{W}(z)\right|+\psi(z) & =\tau\left(\ln \left|F_{W}(z)\right|+\psi_{1}(z)\right)+(1-\tau) \ln \left|\varphi_{2}(z)\right| \\
& \leq \tau\left(d_{1}+H_{L_{1}}(z)+\alpha|z|\right)+(1-\tau)\left(d_{2}+H_{L_{2}}(z)\right) \\
& \leq \tau\left(d_{1}+H_{D}(z)-2 \alpha|z|\right)+(1-\tau)\left(d_{2}+H_{D}(z)+\gamma|z|\right) \\
& \leq d+H_{D}(z)-\tau \alpha|z|, \quad z \in \mathbb{C}^{n},
\end{aligned}
$$

where $d=\tau d_{1}+(1-\tau) d_{2}$. Since the sequence $\left\{K_{j}\right\}$ of compact sets exhausts $D$, from the definition of the support function and the last estimates it easily follows that

$$
\ln \left|F_{W}(z)\right|+\psi(z) \leq d+H_{K_{p}}(z), \quad z \in \mathbb{C}^{n},
$$

for some index $p$. This yields statement 1) of the theorem.

Since $L$ is a compact subset of $D$, by statement 1 ) of Lemma 6.1 we have $\operatorname{Re}\langle y, \xi\rangle=$ $H_{D}(\xi)>H_{L}(\xi)$. Therefore, since the support function of a compact set is continuous, there exists $\delta \in\left(0, \delta^{\prime}\right)$ and $\beta>0$ such that

$$
\operatorname{Re}\langle y, z\rangle>H_{L}(z)+\beta|z|, \quad z \in B(\xi, \delta) .
$$

By (7.5), we also have

$$
(1-\tau) \operatorname{Re}\langle y, z\rangle>H_{K_{m}}(z)+\varepsilon|z| / 2, \quad z \in B(\xi, \delta) .
$$

By (7.7) and (7.9), for some $R>T$ we have

$$
\begin{aligned}
\ln \left|\varphi_{2}(z)\right| & =\ln |\exp \langle y, z\rangle-g(z)| \geq \ln (|\exp \langle y, z\rangle|-|g(z)|) \\
& \geq \ln \left(|\exp \langle y, z\rangle|-\exp \left(d^{\prime}+H_{L}(z)\right)\right) \\
& \geq \operatorname{Re}\langle y, z\rangle+\ln \left(1-\exp \left(d^{\prime}+H_{L}(z)-\operatorname{Re}\langle y, z\rangle\right)\right) \\
& \geq \operatorname{Re}\langle y, z\rangle-\varepsilon|z| / 4, \quad z /|z| \in B(\xi, \delta), \quad|z|>R .
\end{aligned}
$$

Together with (7.10), this implies that

$$
\begin{aligned}
(1-\tau) \ln \left|\varphi_{2}(z)\right| & \geq(1-\tau) \operatorname{Re}\langle y, z\rangle-(1-\tau) \varepsilon|z| / 4 \\
& \geq H_{K_{m}}(z)+\varepsilon|z| / 2-\varepsilon|z| / 4=H_{K_{m}}(z)+\varepsilon|z| / 4, \\
& z /|z| \in B(\xi, \delta), \quad|z|>R .
\end{aligned}
$$

Combining this with (7.4) and (7.5), we arrive at

$$
\begin{aligned}
\psi(z)+\ln \left|F_{W}(z)\right| & =\tau\left(\psi_{1}(z)+\ln \left|F_{W}(z)\right|\right)+(1-\tau) \ln \left|\varphi_{2}(z)\right| \\
& \geq\left(-\tau a_{0}+\varepsilon / 4\right)|z|+H_{K_{m}}(z) \geq H_{K_{m}}(z), \\
& z /|z| \in B(\xi, \delta), \quad|z|>R .
\end{aligned}
$$

Thus, statement 2) is also fulfilled and the theorem follows. 


\section{$\S 8$. Criteria of CONTINUABility}

In this section we show that the conditions presented above that are necessary or sufficient for the analytic continuability of all functions in principal invariant subspaces are in fact equivalent. Therefore, they provide us with a continuability criterion. The conditions given in Theorems 4.1 and 7.1 will be "matched" with the help of the results of $\S 5$ and $\S 6$.

So, we state and prove a criterion for the analytic continuability of functions in principal invariant subspaces on convex domains in $\mathbb{C}^{n}$. More precisely, we present several criteria at once.

Theorem 8.1. Let $D$ be a convex domain in $\mathbb{C}^{n}$, and let $W$ be a principal invariant subspace of $H(D)$ that admits spectral synthesis. Suppose $G$ is a convex subdomain of $D(W)$ containing $D$ and such that the set $\mathbb{S} \cap \Xi \backslash$ int $\Theta_{D}$ is closed. The following statements are equivalent.

a) Every function in $W$ admits analytic continuation to $G$ and, on $G$, can be approximated by linear combinations of elements of $E(W)$.

b) For every $\varsigma \in \mathbb{S} \backslash \Xi$ and every index $m$, there exists a plurisubharmonic function $\psi_{\varsigma, m}$ such that

$$
h_{u_{\varsigma, m}}(z)<H_{D}(z), z \in \mathbb{C}^{n} \backslash\{0\}, \quad \underline{h}_{u_{\varsigma, m}}(\varsigma) \geq H_{K_{m}}(\varsigma),
$$

where $u_{\varsigma, m}=\ln \left|F_{W}\right|+\psi_{\varsigma, m}$.

c) For every compact set $S_{0} \subset \mathbb{S} \backslash \Xi$ and every index $m$, there exists a plurisubharmonic function $\psi$ on $\mathbb{C}^{n}$, a neighborhood $\Omega$ of $S_{0}$, and a constant $T>0$ such that

1) $h_{u}(z)<H_{D}(z), \quad z \in \mathbb{C}^{n} \backslash\{0\}$, where $u=\ln \left|F_{W}\right|+\psi$;

2) $u(z) \geq H_{K_{m}}(z)$ for all $z \in \mathbb{C}^{n}$ with $z /|z| \in \Omega,|z| \geq T$.

d) For every neighborhood $V$ of $\mathbb{S} \cap \Xi \backslash$ int $\Theta_{D}$ there exists a neighborhood $V_{1}$ of the same set such that $V_{1}$ is compactly included in $V$ and the following is true: for every index $m$ there is a neighborhood $V^{\prime}$ of $\mathbb{S} \cap \Xi \backslash$ int $\Theta_{D}$ included in $V_{1}$ and such that for every $\varsigma \in\left(\partial V^{\prime} \backslash \Theta_{D}\right) \cap \mathbb{S}$ there exists a plurisubharmonic function $\psi_{\varsigma, m}$ satisfying the conditions

$$
h_{u_{\varsigma, m}}(z)<H_{D}(z), z \in \mathbb{C}^{n} \backslash\{0\}, \quad \underline{h}_{u_{\varsigma, m}}(\varsigma) \geq H_{K_{m}}(\varsigma),
$$

where $u_{\varsigma, m}=\ln \left|F_{W}\right|+\psi_{\varsigma, m}$.

Remark. Statements b) and c) constitute the essence of Theorem 8.1. The criteria they give can be called, respectively, "local" and "global". Statement d) is a "technical" criterion. On the one hand, it is an easy consequence of b). On the other hand, it is plotted so as to ensure a most easy way from it to statement a) via Theorem 4.1. Clearly, the requirement imposed on $D$ in Theorem 8.1 (that the set $\mathbb{S} \cap \Xi \backslash$ int $\Theta_{D}$ be closed) makes it possible to simplify statement d). But in that case Theorem 4.1 would have also required a revision implied by this restriction on $D$ (in order to make statement d) to imply the assumptions of Theorem 4.1). Thus, the formal simplification in statement d) would have raised the complexity level of the proof of $d) \Rightarrow a$ ), which is already complex as it is.

Proof. a) $\Rightarrow$ b). We fix $\varsigma \in \mathbb{S} \backslash \Xi$ and an index $m$. If a) is true, Theorem 7.1 implies the existence of a plurisubharmonic function $\psi$, an index $p$, and some numbers $d, R, \delta>0$ such that statements 1) and 2) of that theorem are fulfilled. Put $\psi_{\varsigma, m}=\psi$. By inequality 1) in Theorem 7.1 and the definition of the upper indicator, we obtain $h_{u_{\varsigma, m}}(z) \leq H_{K_{p}}<H_{D}(z), z \in \mathbb{C}^{n} \backslash\{0\}$. On the other hand, from inequality 2) and the mean value inequality for subharmonic functions it follows that $\underline{h}_{u_{\varsigma, m}}(\varsigma) \geq H_{K_{m}}(\varsigma)$. Thus, b) is fulfilled. 
b) $\Rightarrow$ c). The inclusion $G \subset D(W)$, the definitions of the sets $\Xi$ and $\Theta_{D}$, and identity (3.1) imply the inclusion $\Delta(W) \cup \Theta_{D} \subset \Xi$. Therefore, every compact subset $S_{0}$ of $\mathbb{S} \backslash \Xi$ does not intersect $\Delta(W) \cup \Theta_{D}$. It remains to apply Proposition 5.3.

c) $\Rightarrow$ d). Fix $\varsigma \in \mathbb{S} \backslash \Xi$ and an index $m$, and put $S_{0}=\{\varsigma\}$. By c), there exists a plurisubharmonic function $\psi$ possessing properties 1) and 2) in c). Let $\psi_{\varsigma, m}=\psi$. Then, as in the proof of the first implication, property 1) yields the first inequality in b) and property 2) yields the second.

b) $\Rightarrow$ d). Let $V$ be an arbitrary compact neighborhood of the set $\mathbb{S} \cap \Xi \backslash \operatorname{int} \Theta_{D}$. This set is closed by assumption. Therefore, it has a neighborhood $V_{1}$ compactly included in $V$. We fix $m$ and put $V^{\prime}=V_{1}$. It remains to observe that an arbitrary point $\varsigma \in\left(\partial V^{\prime} \backslash \Theta_{D}\right) \cap \mathbb{S}$ belongs to $\mathbb{S} \backslash \Xi$.

d) $\Rightarrow$ a). Suppose d) is true. It suffices to show that all assumptions of Theorem 4.1 are fulfilled in this case. Let $V$ be an arbitrary neighborhood of $\Xi \cap \mathbb{S}$. Then $V$ is also a neighborhood of $\mathbb{S} \cap \Xi \backslash$ int $\Theta_{D}$. We begin with constructing a compact set $X \subset V$ the existence of which is required in Theorem 4.1.

Let $V_{1}$ be a neighborhood of $\mathbb{S} \cap \Xi \backslash$ int $\Theta_{D}$ as in statement d) such that $V_{1}$ is compactly included in $V$. Since $\Delta(W)$ is closed, $\Delta(W) \backslash V_{1}$ is also closed. By assumption, $G \subset$ $D(W)$. Therefore (3.1) shows that $\Delta(W)$ is included in $\Xi$. Consequently, $\Delta(W) \backslash V_{1} \subset$ $\operatorname{int} \Theta_{D}$. Let $V_{2}$ be a neighborhood of $\Delta(W) \backslash V_{1}$ compactly included in $\operatorname{int} \Theta_{D}$. Then $V_{3}=V_{1} \cup V_{2}$ is a neighborhood of $\Delta(W)$, which is compactly included in $V$ because $\operatorname{int} \Theta_{D} \subset V$. We choose $\tau>0$ in such a way that the $\tau$-blowup $V_{3}^{\tau}$ is still compactly included in $V$, and put $X=\overline{V_{3}^{\tau}}$.

Fix $\varepsilon>0$ and an index $m$. We must construct an open set $U$ and a plurisubharmonic function $\psi(z)$ on $\mathbb{C}^{n}$ and choose a constant $R>0$ and an index $p$ such that i)-iv) in Theorem 4.1 are true. We start with the construction of $\psi(z)$. Let $V^{\prime}$ be a neighborhood of $\mathbb{S} \cap \Xi \backslash$ int $\Theta_{D}$ as in d) that lies in $V_{1}$. As above, we find a neighborhood $V^{\prime \prime}$ of $\Delta(W) \backslash V^{\prime}$ that is included compactly in int $\Theta_{D}$. Then $V_{0}=V^{\prime} \cup V^{\prime \prime}$ is also a neighborhood of $\Delta(W)$, like $V_{3}$. Also, clearly we may assume that $V^{\prime \prime}$ (and, with it, $V_{0}$ ) is included in $V_{3}$.

By Lemma 6.2, the set $S_{0}=\left(\partial V^{\prime} \backslash \Theta_{D}\right) \cap \mathbb{S}=\left(\partial V_{0} \backslash \Theta_{D}\right) \cap \mathbb{S}$ is compact. As has already been mentioned, $S_{0}$ is a subset of $\mathbb{S} \backslash \Xi$; therefore, it has no points in common with $\Delta(W) \cup \Theta_{D}$. By Proposition 5.3, there exists a function $\psi_{1}$ plurisubharmonic in $\mathbb{C}^{n}$, a neighborhood $\Omega_{0}$ of $S_{0}$, and a constant $T_{1}>0$ such that

$$
h_{u_{1}}(z)<H_{D}(z), \quad z \in \mathbb{C}^{n} \backslash\{0\},
$$

where $u_{1}=\ln \left|F_{W}\right|+\psi_{1}$, and

$$
u_{1}(z) \geq H_{K_{m}}(z) \quad \text { for all } z \text { with } z /|z| \in \Omega_{0}, \quad|z| \geq T_{1} .
$$

Put $S_{1}=\left(\partial V_{0} \backslash \Omega_{0}\right) \cap \mathbb{S}$. Then $S_{1}$ is a compact subset of int $\Theta_{D}$. Indeed, since $\Omega_{0}$ is a neighborhood of $S_{0}$, the definition of $S_{0}$ implies $S_{1} \subset \Theta_{D}$. Suppose $S_{1} \cap \partial \Theta_{D}$ is nonempty, and let $\xi \in S_{1} \cap \partial \Theta_{D}$. Two cases may occur: $\xi \in \Theta_{D}$ and $\xi \notin \Theta_{D}$. If $\xi \in \Theta_{D}$, then $\xi \in\left(\Xi \backslash \operatorname{int} \Theta_{D}\right) \cap \mathbb{S} \subset V^{\prime}$, which is impossible because $\xi \in \partial V_{0}$. But if $\xi \notin \Theta_{D}$, then $\xi \in S_{0} \subset \Omega_{0}$, which is also impossible by the definition of $S_{1}$.

The compact set $S_{1}$ has no points in common with $\Delta(W)$ because $V_{0}$ is a neighborhood of $\Delta(W)$. Let $f$ be a nonzero element of $I_{W} \subset P_{D}$. Then $f$ is divisible by $F_{W}$ and

$$
\ln |f(z)| \leq d_{1}+H_{L}(z), \quad z \in \mathbb{C}^{n}
$$

where $L$ is a compact convex subset of $D$, and $d_{1}$ is a positive constant. By Proposition 5.1, for every $\alpha>0$ there exists a plurisubharmonic function $\psi_{2}$, a neighborhood $\Omega_{1}$ of $S_{1}$, and constants $a_{0}, T_{2}>0$ such that

$$
\begin{aligned}
& u_{2}(z) \geq-a_{0}|z| \quad \text { for all } z \quad \text { with } z /|z| \in \Omega_{1}, \quad|z| \geq T_{2}, \\
& u_{2}(z) \leq d_{1}+H_{L}(z)+\alpha|z|, \quad z \in \mathbb{C}^{n},
\end{aligned}
$$


where $u_{2}=\ln \left|F_{W}\right|+\psi_{2}$. We choose a positive $\alpha$ such that the $\alpha$-blowup $L^{\alpha}$ is compactly included in $D$. Then the last inequality implies that

$$
h_{u_{2}}(z) \leq H_{D}(z), \quad z \in \mathbb{C}^{n} \backslash\{0\} .
$$

Since $S_{1}$ is a compact subset of int $\Theta_{D}$ and $\mathbb{C}^{n} \backslash \Theta_{D}$ is a convex set, Lemma 6.3 shows that there exists a neighborhood $\Omega_{2}$ of $S_{1}$ and a plurisubharmonic function $\psi_{0}$ of order 1 and finite type with the following properties:

$$
\begin{aligned}
& \psi_{0}(z)=0, \quad z \notin \Theta_{D} ; \\
& \psi_{0}(z) \geq A|z| \geq H_{K_{m}}(z)+a_{0}|z|, \quad z /|z| \in \Omega_{2},
\end{aligned}
$$

where $A=\sup _{\xi \in B(0,1)} H_{K_{m}}(\xi)+a_{0}$. We put $\psi_{2}^{\prime}=\psi_{2}+\psi_{0}$ and $\Omega_{2}^{\prime}=\Omega_{1} \cap \Omega_{2}$. By (8.4), (8.5), and the definition of $\Theta_{D}$, we have

$$
h_{u_{2}^{\prime}}(z)<H_{D}(z), \quad z \in \mathbb{C}^{n} \backslash\{0\},
$$

where $u_{2}^{\prime}=\ln \left|F_{W}\right|+\psi_{2}^{\prime}$. Moreover, by (8.3) and (8.6) we obtain

$$
u_{2}^{\prime}(z) \geq H_{K_{m}}(z), \quad z /|z| \in \Omega_{2}^{\prime}, \quad|z| \geq T_{2} .
$$

Let

$$
\psi(z)=\max \left\{\psi_{1}(z), \psi_{2}^{\prime}(z)\right\}+c, \quad z \in \mathbb{C}^{n},
$$

where $c$ is a positive constant to be chosen later. Also put $\Omega=\Omega_{0} \cup \Omega_{2}^{\prime}$ and $T=$ $\max \left\{T_{1}, T_{2}\right\}$. Then, by (8.1) and (8.7), we have

$$
h_{u}(z)<H_{D}(z), \quad z \in \mathbb{C}^{n} \backslash\{0\},
$$

where $u=\ln \left|F_{W}\right|+\psi$. Formulas (8.2) and (8.8) show that

$$
u(z) \geq H_{K_{m}}(z) \quad \text { for all } z \text { with } z /|z| \in \Omega, \quad|z| \geq T .
$$

By (8.9) and Lemma 5.4, we may assume that $\psi(z)$ is a continuous plurisubharmonic function.

Now, we pass to the construction of the open set $U$ whose existence is required in Theorem 4.1. As has already been mentioned, $V_{0}$ is a neighborhood of $\Delta(W)$. By the definition of $\Delta(W)$, there exists $R^{\prime}>0$ such that the set $N(W) \backslash V_{0}^{\prime}$ is included in the ball $B\left(0, R^{\prime}\right)$. Here $V_{0}^{\prime}$ is the cone with vertex at the origin generated by $V_{0} \cap \mathbb{S}$, i.e., $V_{0}^{\prime}=\left\{z: z=t \xi, \xi \in V_{0} \cap \mathbb{S}, t>0\right\}$. We put $U=V_{0}^{\prime} \cup B\left(0, R^{\prime}\right)$. By the choice of $R^{\prime}$, item i) in Theorem 4.1 is fulfilled for $U$. The definition of $X$ and $V_{0}^{\prime}$ implies that for some $R>R^{\prime}$ and every $z \in U[\varepsilon] \backslash B(0, R)$ the point $z /|z|$ belongs to $X$. This yields item ii). Furthermore, for all $z \in U[\varepsilon] \backslash(U \cup B(0, T))$ with sufficiently large absolute value, the point $z /|z|$ belongs to $\Omega$. Thus, (8.10) (and, with it, item iii)) is fulfilled for such a $z$. In order to ensure iii) on the remaining (bounded) part of $U[\varepsilon] \backslash U$, we only need to choose $c>0$ in the definition of $\psi(z)$ appropriately. This can be done because the function $\psi(z)$ is continuous and $F_{W}$ has no zeros outside $U$, implying that $\ln \left|F_{W}\right|$ is continuous outside $U$. Finally, increasing $R$ in (8.9) if necessary, it is easy to ensure iv) with some index $p$ (as in the Introduction, it suffices to employ the Hartogs theorem on the upper limit for families of subharmonic functions). This finishes the proof of the theorem.

Remarks. 1. For the set $\mathbb{S} \cap \Xi \backslash$ int $\Theta_{D}$ to be closed, it suffices that $H_{D}(z)$ be continuous on this set (this is not a necessary condition). (Indeed, let a sequence $\left\{z_{k}\right\} \subset \mathbb{S} \cap \Xi \backslash \operatorname{int} \Theta_{D}$ converge to $z_{0}$. Since $H_{D}$ is continuous and $H_{G}$ is lower semicontinuous, the inclusion $D \subset G$ implies $H_{G}\left(z_{0}\right) \geq H_{D}\left(z_{0}\right)=\lim _{k \rightarrow \infty} H_{D}\left(z_{k}\right)=\lim _{k \rightarrow \infty} H_{G}\left(z_{k}\right) \geq H_{G}\left(z_{0}\right)$.) For example, this is the case if $D$ is a bounded domain. On the complex plane, the set $\mathbb{S} \cap \Xi \backslash$ int $\Theta_{D}$ is always closed; see [26, Lemma 7].

2. The proof of the implication $\mathrm{d}$ ) $\Rightarrow$ a) shows that the assumptions of Theorem 4.1 constitute yet another statement equivalent to those in Theorem 8.1. 
3. If the set $\mathbb{S} \cap \Xi \backslash \operatorname{int} \Theta_{D}$ is empty (in which case necessarily $\Delta(W) \subset \operatorname{int} \Theta_{D}$ by (3.1) and Lemma 3.3), d) involves no restrictions. Thus, under this condition, the functions in the invariant subspace extend to $G$ without any additional assumptions. Furthermore, the proof of the implication d) $\Rightarrow$ a) simplifies. The function $\psi_{1}$ is not needed any longer. It suffices to put $V_{0}=V^{\prime \prime}$, where $V^{\prime \prime}$ is a neighborhood of $\Delta(W)$ compactly included in int $\Theta_{D}$, and define $S_{1}=\partial V_{0} \cap \mathbb{S}$. After that, $\psi$ is defined by the formula $\psi(z)=\psi_{2}^{\prime}(z)+c$. Even a statement stronger than the implication d) $\Rightarrow$ a) holds true.

Corollary 8.1. Let $D$ be a convex domain in $\mathbb{C}^{n}$, and let $W$ be a principal invariant subspace of $H(D)$ that admits spectral synthesis. Suppose that $\Delta(W) \subset \operatorname{int} \Theta_{D}$. Then every function belonging to $W$ extends up to an entire function and can be approximated on $\mathbb{C}^{n}$ by linear combinations of elements of $E(W)$.

Proof. The inclusion $\Delta(W) \subset$ int $\Theta_{D}$ means that $D(W)$ coincides with $\mathbb{C}^{n}$. Put $G=\mathbb{C}^{n}$. Then $\Xi=\Theta_{D}$, implying that the set $\mathbb{S} \cap \Xi \backslash \operatorname{int} \Theta_{D}$ is empty. Nevertheless, all assumptions of Theorem 4.1 are satisfied. This can be proved as the implication d) $\Rightarrow$ a) in Theorem 8.1 with the simplifications indicated in Remark 3. Then the corollary follows from the claim of Theorem 4.1.

Corollary 8.2. Let $D$ be a convex domain in $\mathbb{C}^{n}$, and let $W$ be a principal invariant subspace of $H(D)$ that admits spectral synthesis. Suppose that $\Theta_{D}$ is an open set. Then $\Delta(W) \subset \Theta_{D}$ is a necessary and sufficient condition for every function in $W$ to be extendible up to an entire function and to be approximable on $\mathbb{C}^{n}$ by linear combinations of elements of $E(W)$.

Proof. This follows from Lemma 3.3 and Corollary 8.1.

\section{§9. Approximation of PLURISUbharmonic FUnCTIONS}

In all preceding papers on the continuation problem, conditions of extendibility of functions belonging to an invariant subspace $W$ were formulated in terms of the existence of a certain entire function in $I_{W}$ with appropriate lower asymptotic estimates (in the particular case where $W$ is the space of solutions of a homogeneous convolution equation, the role of such a function was played by the characteristic function of the convolution operator). We shall also present some conditions in this traditional form. But before that we prove an auxiliary result about approximation of plurisubharmonic functions by the logarithm of the modulus of an entire function; it is based on Yulmukhametov's work in 39 .

Lemma 9.1. Let $F$ be an entire function in $\mathbb{C}^{n}$ of order at most 1 (and not necessarily of finite type at order 1 ), and let $\psi$ be a plurisubharmonic function. Suppose we are given a number $b>0$ and a compact convex set $L \subset \mathbb{C}^{n}$ such that

$$
u(z) \leq d+H_{L}(z), \quad z \in \mathbb{C}^{n},
$$

where $u=\ln |F|+\psi$. Then there exists an entire function $\varphi$ of exponential type with the following properties:

1) $\varphi / F$ is an entire function;

2) $h_{\varphi}(z) \leq H_{L}(z), z \in \mathbb{C}^{n}$

3) for every $\varsigma \in \mathbb{S}$ there exists a sequence $\left\{z_{k}\right\} \in \Phi_{0}(\varsigma)$ such that

$$
\lim _{k \rightarrow \infty}\left|z_{k}\right|^{-1}|\ln | \varphi\left(z_{k}\right)\left|-u\left(z_{k}\right)\right|=0 .
$$

Proof. By assumption, there exist constants $A, B, C, D>0$ such that

$$
\psi(z)+\ln |F(z)| \leq A+B|z|^{\rho}, \quad \ln |F(z)| \leq C+D|z|^{\rho},
$$


for $\rho=1+1 / 16$ and all $z \in \mathbb{C}^{n}$. By Lemma 5.2, we have

$$
\psi(z) \leq a+b|z|^{\rho}, \quad z \in \mathbb{C}^{n},
$$

for some $a, b>0$. Therefore, Theorem 3 in 39 applies to $\psi$, yielding an entire function $\tilde{\varphi}$ with the property

$$
|\ln | \widetilde{\varphi}(z)|-\psi(z)| \leq c(1+|z|)^{4 / 5+1 / 10}, \quad z \in \mathbb{C}^{n} \backslash E,
$$

where $E$ is a subset of $\mathbb{C}^{n}$ that can be covered by a system of balls $B\left(y_{i}, r_{i}\right), i \geq 1$, such that $r_{i} \leq\left|y_{i}\right| / 2, i \geq 1$, and

$$
\sum_{R / 2 \leq\left|y_{i}\right| \leq R} r_{i}^{2 n-1} \leq \beta \frac{R^{2 n-1}}{\ln (R+e)}, \quad R>0,
$$

where $\beta$ and $c$ are some positive constants (the estimate $r_{i} \leq\left|y_{i}\right| / 2$ was not mentioned in the statement cited, but arose in the proof). Next, by (16) in [39, $\widetilde{\varphi}$ obeys the upper estimate

$$
\ln |\widetilde{\varphi}(z)| \leq \psi^{\prime}(z)+c_{1}(1+|z|)^{2 \gamma / 3}, \quad z \in \mathbb{C}^{n},
$$

where $c_{1}>0, \gamma=(7 / 5) \rho$, and

$$
\psi^{\prime}(z)=\int_{B(0,1)} \psi\left(z+(1+|z|)^{1-\rho / 5} \xi\right) \alpha\left(|\xi|^{2}\right) d \sigma(\xi),
$$

here $\alpha(t)$ is a nonnegative infinitely differentiable function on the real line vanishing outside of $(0,1)$ and satisfying

$$
\omega_{n} \int_{1}^{0} \alpha\left(t^{2}\right) t^{2 n-1} d t=1
$$

where $\omega_{n}$ is the area of the unit sphere in $\mathbb{C}^{n}=\mathbb{R}^{2 n}$.

We put $\varphi=F \widetilde{\varphi}$. Then $\varphi / F$ is an entire function. We show that $\varphi$ is of exponential type. Fix $z \in \mathbb{C}^{n}$. By the mean value inequality for the subharmonic function $q(\xi)=$ $\ln \left|F\left(z+(1+|z|)^{1-\rho / 5} \xi\right)\right|$, we have

$$
\ln |F(z)|=q(0) \leq \frac{1}{\omega_{n} t^{2 n-1}} \int_{\partial B(0, t)} q(\xi) d \omega(\xi), \quad t>0,
$$

where $d \omega$ is the standard surface measure in $\mathbb{C}^{n}=\mathbb{R}^{2 n}$. Combining this with (9.5), we obtain

$$
\ln |F(z)| \leq \int_{B(0,1)} q(\xi) \alpha\left(|\xi|^{2}\right) d \sigma(\xi) .
$$

Together with (9.3) and (9.4), this implies that

$$
\begin{aligned}
\ln |\varphi(z)| \leq & \psi^{\prime}(z)+c_{1}(1+|z|)^{2 \gamma / 3}+\int_{B(0,1)} q(\xi) \alpha\left(|\xi|^{2}\right) d \sigma(\xi) \\
= & \int_{B(0,1)} \psi\left(z+(1+|z|)^{1-\rho / 5} \xi\right) \\
& \quad+\ln \left|F\left(z+(1+|z|)^{1-\rho / 5} \xi\right)\right| \alpha\left(|\xi|^{2}\right) d \sigma(\xi)+c_{1}(1+|z|)^{2 \gamma / 3} \\
= & \int_{B(0,1)} u\left(z+(1+|z|)^{1-\rho / 5} \xi\right) \alpha\left(|\xi|^{2}\right) d \sigma(\xi)+c_{1}(1+|z|)^{2 \gamma / 3}, \quad z \in \mathbb{C}^{n} .
\end{aligned}
$$

Let $M>0$ be such that $\sup _{w \in S} H_{L}(w) \leq M$; then

$$
H_{L}(w) \leq M|w|, \quad w \in \mathbb{C}^{n} .
$$


By the above and the upper estimate for $u$ in the assumptions of the lemma, we obtain

$$
\begin{array}{r}
\ln |\varphi(z)| \leq\left(d+\sup _{w \in B\left(z,(1+|z|)^{1-\rho / 5}\right)} M|w|\right) \int_{B(0,1)} \alpha\left(|\xi|^{2}\right) d \sigma(\xi)+c_{1}(1+|z|)^{2 \gamma / 3}, \\
z \in \mathbb{C}^{n} .
\end{array}
$$

Since $1-\rho / 5<1$ and $2 \gamma / 3=14 \rho / 15<1$, it follows that

$$
\ln |\varphi(z)| \leq a^{\prime}+b^{\prime}|z|, \quad z \in \mathbb{C}^{n},
$$

where $a^{\prime}$ and $b^{\prime}$ are positive constants. Thus, $\varphi$ is an entire function of exponential type, and the definition of the indicator shows that $h_{\varphi}(z) \leq b^{\prime}|z|, z \in \mathbb{C}^{n}$. We show that statement 2) in the lemma holds true. Suppose that

$$
h_{\varphi}\left(z_{0}\right)>H_{L}\left(z_{0}\right)
$$

for some $z_{0}$. Since the indicator and the support function are positive homogeneous, we may assume that $z_{0} \in \mathbb{S}$. Since the support function of a bounded set is continuous, we can choose $\varepsilon, \delta^{\prime}>0$ such that

$$
h_{\varphi}\left(z_{0}\right)>H_{L}(z)+19 \varepsilon, \quad z \in B\left(z_{0}, 4 \delta^{\prime}\right) .
$$

By the definition of $h_{\varphi}$, there exists a sequence $\left\{x_{k}\right\}$ convergent to $z_{0}$ and an unbounded and monotone increasing sequence $\left\{t_{k}\right\}$ of positive number with

$$
\ln \left|\varphi\left(t_{k} x_{k}\right)\right| / t_{k} \geq h_{\varphi}\left(z_{0}\right)-\varepsilon, \quad k=1,2, \ldots
$$

The properties of indicators (see, e.g., [1]) imply the existence of constants $\delta \in\left(0, \delta^{\prime}\right)$ and $T>0$ such that

$$
\ln |\varphi(t z)| / t \leq h_{\varphi}\left(z_{0}\right)+\varepsilon, \quad z \in B\left(z_{0}, 8 e \delta\right), t \geq T .
$$

Clearly, we may assume that $\delta<1 / 30$. We choose $k_{0}$ such that $\left|x_{k}-z_{0}\right|<\delta, t_{k} \geq T$, and $t_{k}>R_{0}$ for all $k \geq k_{0}$, where $R_{0}>0$ satisfies the inequality $\beta / \ln \left(R_{0}+e\right)<(\delta / 6)^{2 n-1}$. Fix $k \geq k_{0}$ and denote by $J_{k}$ the set of all indices $i$ for which the ball $B\left(y_{i}, r_{i}\right)$ intersects $B\left(t_{k} x_{k}, 3 \delta t_{k}\right) \backslash B\left(t_{k} x_{k}, 2 \delta t_{k}\right)$. Let $M_{i}, i \in J_{k}$, be the area of the central projection $S_{i}$ of the ball $B\left(y_{i}, r_{i}\right)$ to the sphere $S\left(t_{k} x_{k}, 3 \delta t_{k}\right)\left(S_{i}\right.$ consists of the points $\xi \in S\left(t_{k} x_{k}, 3 \delta t_{k}\right)$ such that the ray $\lambda=t_{k} x_{k}+\alpha\left(\xi-t_{k} x_{k}\right), \alpha>0$, intersects $\left.B\left(y_{i}, r_{i}\right)\right)$.

We show that the total area $\sum_{i \in J_{k}} M_{i}$ of these projections is smaller than the area of the sphere $S\left(t_{k} x_{k}, 3 \delta t_{k}\right)$. Let $i \in J_{k}$. Since $r_{i} \leq\left|y_{i}\right| / 2, \delta<1 / 30$, and the balls $B\left(y_{i}, r_{i}\right)$ and $B\left(t_{k} x_{k}, 3 \delta t_{k}\right)$ intersect, we have

$$
\frac{3\left|y_{i}\right|}{2} \geq(1-3 \delta) t_{k} \geq \frac{9}{10} t_{k} \geq \frac{9}{10} R_{0} .
$$

By the choice of $R_{0}$, for $R=2\left|y_{i}\right|>R_{0}$ we obtain $r_{i} \leq \delta\left|y_{i}\right| / 3$. Now, on the one hand, since the intersection $B\left(y_{i}, r_{i}\right) \cap B\left(t_{k} x_{k}, 3 \delta t_{k}\right)$ is nonempty, it follows that

$$
88\left|y_{i}\right| / 90 \leq(1-\delta / 3)\left|y_{i}\right| \leq(1+3 \delta) t_{k} \leq 11 t_{k} / 10 ;
$$

on the other hand, denoting by $w$ a point in common for $B\left(y_{i}, r_{i}\right)$ and $B\left(t_{k} x_{k}, 3 \delta t_{k}\right) \backslash$ $B\left(t_{k} x_{k}, 2 \delta t_{k}\right)$, for every $z \in B\left(y_{i}, r_{i}\right)$ we obtain

$$
\begin{aligned}
\left|z-t_{z} x_{k}\right| & =\left|w-t_{k} x_{k}\right|-|z-w| \geq 2 \delta t_{k}-2 r_{i} \\
& \geq 2 \delta t_{k}-2 \delta\left|y_{i}\right| / 3 \geq 2 \delta t_{k}-2 \cdot 9 \delta t_{k} /(8 \cdot 3)>\delta t_{k} .
\end{aligned}
$$

This means that the ball $B\left(y_{i}, r_{i}\right)$ lies outside of the ball $B\left(t_{k} x_{k}, \delta t_{k}\right)$. Therefore, the area $M_{i}$ of the projection of $B\left(y_{i}, r_{i}\right)$ to the sphere $S\left(t_{k} x_{k}, 3 \delta t_{k}\right)$ does not exceed the surface area of the ball of radius $3 r_{i}$, i.e.,

$$
M_{i} \leq \omega_{n}\left(3 r_{i}\right)^{2 n-1}, \quad i \in J_{k} .
$$


By (9.10) and (9.11), all numbers $\left|y_{i}\right|, i \in J_{k}$, belong to the interval $\left[3 t_{k} / 5,9 t_{k} / 8\right]$, which is included in $\left[9 t_{k} / 16,9 t_{k} / 8\right]$. By $(9.2)$ and the choice of $R_{0}$ and $k_{0}$, for $R=9 t_{k} / 8$ we obtain

$$
\sum_{i \in J_{k}} M_{i} \leq \omega_{n} \sum_{R / 2 \leq\left|y_{i}\right| \leq R}\left(3 r_{i}\right)^{2 n-1} \leq \omega_{n}\left(\frac{9 \delta t_{k}}{8 \cdot 6}\right)^{2 n-1} \leq \omega_{n}\left(3 \delta t_{k}\right)^{2 n-1} .
$$

Therefore, the total area of the projections $S_{i}, i \in J_{k}$, is smaller than the area of the sphere $S\left(t_{k} x_{k}, 3 \delta t_{k}\right)$, and we can choose $\varsigma_{k} \in \mathbb{S}$ such that the point $\left(t_{k} x_{k}+3 \delta t_{k} \varsigma_{k}\right) \in$ $S\left(t_{k} x_{k}, 3 \delta t_{k}\right)$ does not belong to any of $S_{i}, i \in J_{k}$. By construction, the interval $\left(\xi_{k}^{\prime}, \xi_{k}\right)=\left\{\xi=t_{k} x_{k}+\alpha \varsigma_{k}: \alpha \in\left(2 \delta t_{k}, 3 \delta t_{k}\right)\right\}$ does not intersect the balls $B\left(y_{i}, r_{i}\right)$, $i \in J_{k}$; consequently, it does not intersect $E$. Now we show that $\varphi(z)$ admits appropriate lower estimates at a point of this interval. To do this, we consider the function $f(z)=\varphi(z) / \varphi\left(t_{k} x_{k}\right)$. Formulas (9.8) and (9.9) show that

$$
\ln |f(z)| \leq 2 \varepsilon t_{k}, \quad z \in B\left(t_{k} x_{k}, 6 e \delta t_{k}\right) \subset B\left(t_{k} z_{0}, 8 e \delta t_{k}\right) .
$$

By Lemma 5.1, for $\eta=1 / 36$ we can find $\tau_{k} \in\left(2 \delta t_{k}, 3 \delta t_{k}\right)$ such that

$$
\ln \left|f\left(w_{k}\right)\right| \geq-(4-\ln \eta) 2 \varepsilon t_{k} \geq-16 \varepsilon t_{k},
$$

where $w_{k}=t_{k} x_{k}+\tau_{k} \varsigma_{k}$. By (9.8), (9.7), the fact that $w_{k} \in B\left(t_{k} z_{0}, 4 \delta t_{k}\right)$ for $k \geq k_{0}$, and the homogeneity of the support function, we have

(9.12) $\ln \left|\varphi\left(w_{k}\right)\right| \geq\left(h_{\varphi}\left(z_{0}\right)-17 \varepsilon\right) t_{k} \geq\left(H_{L}\left(w_{k} / t_{k}\right)+2 \varepsilon\right) t_{k}=H_{L}\left(w_{k}\right)+2 \varepsilon t_{k}, \quad k \geq k_{0}$.

On the other hand, since $w_{k}$ belongs to the interval $\left(\xi_{k}^{\prime}, \xi_{k}\right)$ and, therefore, does not belong to $E$, by (9.1) and the estimate for $u$ in the assumptions of the lemma we obtain

$$
\begin{aligned}
\ln \left|\varphi\left(w_{k}\right)\right| & \leq u\left(w_{k}\right)+c\left(1+\left|w_{k}\right|\right)^{9 / 10} \\
& \leq d+H_{L}\left(w_{k}\right)+c\left(1+\left|w_{k}\right|\right)^{9 / 10} \leq H_{L}\left(w_{k}\right)+\varepsilon\left|w_{k}\right|
\end{aligned}
$$

for $k$ sufficiently large. This contradicts (9.12) because

$$
\left|w_{k}\right| \leq t_{k}\left|z_{0}\right|+4 \delta t_{k} \leq(1+2 / 15) t_{k} .
$$

Thus, (9.6) is false and statement 2) is proved. It remains to show that 3 ) is also true. Arguing much as above (in the discussion of the projections of the balls $B\left(y_{i}, r_{i}\right)$ to the sphere), it is easy to show that for every $m \geq 1$ there exists a number $R(m)>0$ such that, for every $z$ with $|z| \geq R(m)$, the sphere $S(z,|z| / m)$ contains a point $w$ not belonging to $E$. Fix $\varsigma \in \mathbb{S}$ and $m \geq 1$. For every $k$ with $R(m) \leq k<R(m+1)$, choose $z_{k} \in S(k \varsigma, k / m)$ that does not belong to $E$. It can easily be seen that the resulting sequence belongs to $\Phi_{0}(\varsigma)$. But

$$
\lim _{k \rightarrow \infty}\left|z_{k}\right|^{-1}|\ln | \varphi\left(z_{k}\right)\left|-u\left(z_{k}\right)\right|=0
$$

by (9.1). This proves the lemma.

\section{$\S 10$. OTHER CRITERIA OF CONTINUABILITY}

As before, let $D$ be a convex domain and $\left\{K_{m}\right\}$ a sequence of compact convex sets exhausting it. Suppose $W$ is a nontrivial invariant subspace of $H(D)$ that admits spectral synthesis. For every index $m$, we introduce the function

$$
\psi_{m}(z)=\limsup _{\xi \rightarrow z} \sup \left\{\ln |\varphi(\xi)|-\ln \left|F_{W}(\xi)\right|: \varphi \in I_{W}, \ln |\varphi(y)| \leq H_{K_{m}}(y) \text { for all } y\right\} .
$$

The functions $\ln |\varphi(\xi)|-\ln \left|F_{W}(\xi)\right|$ are plurisubharmonic (because $\varphi$ belongs to $I_{W}$ and, therefore, is divisible by $F_{W}$ ), and the family in braces is bounded on every compact set by Lemma 5.2. Consequently, $\psi_{m}$ is a plurisubharmonic function. 
Theorem 10.1. Let $D$ be a convex domain in $\mathbb{C}^{n}$ and $W$ a principal invariant subspace of $H(D)$ admitting spectral synthesis. Suppose $G$ is a convex subdomain of $D(W)$ including $D$ and such that the set $\mathbb{S} \cap \Xi \backslash$ int $\Theta_{D}$ is closed. The following statements are equivalent.

a) Every function in $W$ admits analytic continuation to $G$ and, on $G$, can be approximated by linear combinations of elements of $E(W)$.

b) For every compact set $S_{0} \subset \mathbb{S} \backslash \Xi$ and every index $m$, there exists an entire function $\varphi \in I_{W}$ with

$$
\underline{h}_{\varphi}(\varsigma) \geq H_{K_{m}}(\varsigma), \quad \varsigma \in S_{0} .
$$

c) For every compact set $S_{0} \subset \mathbb{S} \backslash \Xi$ and every index $m$, there exists an index $p$ with

$$
\underline{h}_{u_{p}}(\varsigma) \geq H_{K_{m}}(\varsigma), \quad \varsigma \in S_{0},
$$

where $u_{p}=\ln \left|F_{W}\right|+\psi_{p}$.

Proof. a) $\Rightarrow$ b). Suppose a) is fulfilled. By Theorem 8.1, for every compact set $S_{0} \subset \mathbb{S} \backslash \Xi$ and every index $m$, there exists a plurisubharmonic function $\psi$ on $\mathbb{C}^{n}$, a neighborhood $\Omega$ of $S_{0}$, and a constant $T>0$ such that

$$
\begin{gathered}
h_{u}(z)<H_{D}(z), \quad z \in \mathbb{C}^{n} \backslash\{0\}, \quad \text { where } u=\ln \left|F_{W}\right|+\psi ; \\
u(z) \geq H_{K_{m}}(z) \quad \text { for all } z \text { with } z /|z| \in \Omega, \quad|z| \geq T .
\end{gathered}
$$

The first inequality shows that $u(z) \leq d+H_{L}(z), z \in \mathbb{C}^{n}$, where $d>0$ and $L$ is a compact convex subset of $D$. Then Lemma 9.1 implies the existence of an entire function $\varphi$ of exponential type satisfying 1)-3) in that lemma. Since $W$ admits spectral synthesis, properties 1) and 2) show that $\varphi \in I_{W}$ (see the remark to Lemma 3.1). By property 3), formula (10.2), and the continuity of the support function of a compact set, for every $\varsigma \in S_{0}$ there exists a sequence $\left\{z_{k}\right\} \in \Phi_{0}(\varsigma)$ with

$$
\liminf _{k \rightarrow \infty}\left|z_{k}\right|^{-1} \ln \left|\varphi\left(z_{k}\right)\right| \geq H_{K_{m}}(\varsigma) .
$$

We show that this inequality implies (10.1). Suppose the contrary, i.e., $\underline{h}_{\varphi}(\varsigma)<$ $H_{K_{m}}(\varsigma)-2 \varepsilon$ for some $\varepsilon>0$ and $\varsigma \in S_{0}$. Then, by Proposition 9.3 in [7], there exists $\delta>0$ and a sequence $\left\{t_{j}\right\}_{j=1}^{\infty}$ such that $t_{j} \rightarrow+\infty$ and

$$
\ln \left|\varphi\left(t_{j} \eta\right)\right| / t_{j} \leq H_{K_{m}}(\varsigma)-2 \varepsilon, \quad \eta \in B(\varsigma, \delta), \quad j=1,2, \ldots
$$

Reducing $\delta>0$ if necessary, we easily deduce that

$$
\ln |\varphi(z)| /|z| \leq H_{K_{m}}(\varsigma)-\varepsilon, \quad z \in B\left(t_{j} \varsigma, \delta t_{j}\right), \quad j=1,2, \ldots
$$

Together with (10.3), this means that, for $k$ sufficiently large, none among the $z_{k}$ belongs to any of the balls $B\left(t_{j} \varsigma, \delta t_{j}\right), j=1,2, \ldots$ The definition of $\Phi_{0}(\varsigma)$ enables us to find $k_{0}$ with

$$
\left|z_{k+1}\right|<(1+\delta / 2)\left|z_{k}\right|, \quad\left|z_{k} /\right| z_{k}|-\varsigma|<\delta / 2, \quad k>k_{0} .
$$

Moreover, as was observed when we defined $\Phi_{0}(\varsigma)$, we may assume that the $\left|z_{k}\right|$ increase monotonically and tend to $+\infty$. Therefore, for every $j_{0}$ and every $j>j_{0}$, there exists $k_{j}>k_{0}$ such that $\left|z_{k_{j}}\right| \leq t_{j} \leq\left|z_{k_{j}+1}\right|$ and the points $z_{k_{j}}, z_{k_{j}+1}$ do not belong to $B\left(t_{j} \varsigma, \delta t_{j}\right)$. Using the first inequality in (10.4), we obtain $t_{j} /\left|z_{k_{j}}\right| \leq\left|z_{k_{j}+1}\right| /\left|z_{k_{j}}\right|<$ $1+\delta / 2$. It follows that

$$
\left|z_{k_{j}}\right| \leq t_{j}<\left|z_{k_{j}}\right|+\delta\left|z_{k_{j}}\right| / 2 \leq\left|z_{k_{j}}\right|+\delta t_{j} / 2 .
$$

Taking the second inequality in (10.4) into account, we obtain $\left|z_{k_{j}}-t_{j} \varsigma\right| \leq\left|z_{k_{j}}\right|\left|z_{k_{j}} /\right| z_{k_{j}}|-\varsigma|+|| z_{k_{j}}\left|\varsigma-t_{j} \varsigma\right|<\delta\left|z_{k_{j}}\right| / 2+\delta t_{j} / 2 \leq \delta t_{j} / 2+\delta t_{j} / 2=\delta t_{j}$. This means that $z_{k_{j}}$ belongs to $B\left(t_{j} \varsigma, \delta t_{j}\right)$. This contradiction completes the proof. 
b) $\Rightarrow$ c). Let $\varphi \in I_{W}$ satisfy (10.1). Since $I_{W} \subset P_{D}$, by the definition of $P_{D}$ there exists an index $p$ and a number $c>0$ such that

$$
|\varphi(z)| \leq c \exp H_{K_{p}}(z), \quad z \in \mathbb{C}^{n} .
$$

Now, formula (10.1) and the definitions of $\psi_{p}$ and of the lower indicator imply that

$$
\underline{h}_{u_{p}}(\varsigma) \geq \underline{h}_{\varphi / c}(\varsigma)=\underline{h}_{\varphi}(\varsigma) \geq H_{K_{m}}(\varsigma), \quad \varsigma \in S_{0} .
$$

c) $\Rightarrow$ a). If c) is true, statement b) in Theorem 8.1 is also true. Indeed, it suffices to observe that

$$
h_{u_{p}}(z)<H_{D}(z), \quad z \in \mathbb{C}^{n} \backslash\{0\}
$$

for every index $p$. Now, Theorem 8.1 implies a).

Now we consider the case of a bounded convex domain separately. In this case, simpler criteria of continuability can be given.

Let $D$ be a bounded convex domain, and let $W$ be a nontrivial closed subspace of $H(D)$ that admits spectral synthesis. We introduce the functions

$$
\begin{aligned}
\psi_{0}(z)= & \limsup _{\xi \rightarrow z} \sup \left\{\ln |\varphi(\xi)|-\ln \left|F_{W}(\xi)\right|:\right. \\
& \left.\quad \in H\left(\mathbb{C}^{n}\right), \varphi / F_{W} \in H\left(\mathbb{C}^{n}\right), \ln |\varphi(y)| \leq H_{D}(y) \text { for all } y\right\}, \\
\psi_{0}^{\prime}(z)= & \limsup _{\xi \rightarrow z} \sup \{\psi(\xi): \\
& \left.\psi \text { is plurisubharmonic, } \psi(y)+\ln \left|F_{W}(y)\right| \leq H_{D}(y) \text { for all } y\right\} .
\end{aligned}
$$

These functions are plurisubharmonic on $\mathbb{C}^{n}$ (the verification is much as for $\psi_{m}$ ). Directly from the definitions we deduce that $\psi_{0}(z) \leq \psi_{0}^{\prime}(z), z \in \mathbb{C}^{n}$. It is natural to ask whether equality occurs here. This question requires an additional study, which is beyond the framework of this paper.

Theorem 10.2. Let $D$ be a bounded convex subset of $\mathbb{C}^{n}, W$ a principal invariant subspace of $H(D)$ admitting spectral synthesis, and $G$ a convex subdomain of $D(W)$ containing $D$. The following statements are equivalent.

a) Every function in $W$ admits analytic continuation to $G$ and can be approximated on $G$ by linear combinations of elements of $E(W)$.

b) For every compact set $S_{0} \subset \mathbb{S} \backslash \Xi$ and every index $m$, there exists $R>0$ such that

$$
u_{0}^{\prime}(z) \geq H_{K_{m}}(z) \text { for all } z \text { with } z /|z| \in S_{0}, \quad|z| \geq R
$$

(here $u_{0}^{\prime}=\psi_{0}^{\prime}+\ln \left|F_{W}\right|$ ).

c) There exists an entire function $\varphi$ of exponential type with the following properties:

1) $\varphi$ is divisible by $F_{W}$,

2) $h_{\varphi}(z) \leq H_{D}(z), z \in \mathbb{C}^{n}$,

3) $\underline{h}_{\varphi}(\varsigma)=h_{\varphi}(\varsigma)=H_{D}(\varsigma), \varsigma \in \overline{\mathbb{S} \backslash \Xi}$.

d) $\underline{h}_{u_{0}}(\varsigma)=h_{u_{0}}(\varsigma)=H_{D}(\varsigma), \varsigma \in \overline{\mathbb{S} \backslash \Xi}$, where $u_{0}=\psi_{0}+\ln \left|F_{W}\right|$.

e) There exists a plurisubharmonic function $\psi$ such that

1) $h_{u}(z) \leq H_{D}(z), z \in \mathbb{C}^{n}$, where $u=\psi+\ln \left|F_{W}\right|$,

2) $\underline{h}_{u}(\varsigma)=h_{u}(\varsigma)=H_{D}(\varsigma), \varsigma \in \mathbb{S} \backslash \Xi$.

Proof. a) $\Rightarrow$ b). If a) is true, then, by c) in Theorem 8.1, there exists a function $\psi$ plurisubharmonic on $\mathbb{C}^{n}$ and a constant $T>0$ such that

$$
\begin{aligned}
h_{u}(z) & <H_{D}(z), \quad z \in \mathbb{C}^{n} \backslash\{0\}, \text { where } u=\ln \left|F_{W}\right|+\psi ; \\
u(z) & \geq H_{K_{m+1}}(z) \text { for all } z \text { with } z /|z| \in S_{0}, \quad|z| \geq T .
\end{aligned}
$$


By (10.5), $u(z) \leq c+H_{D}(z), z \in \mathbb{C}^{n}$, for some $c>0$. Then (10.6) and the definition of $\psi_{0}^{\prime}$ imply that

$$
u_{0}^{\prime}(z) \geq u(z)-c \geq H_{K_{m+1}}(z)-c \text { for all } z \text { with } z /|z| \in S_{0}, \quad|z| \geq T .
$$

Since $K_{m}$ is included in the interior of $K_{m+1}$, we have

$$
H_{K_{m+1}}(z)-c \geq H_{K_{m}}(z), \quad|z| \geq R,
$$

for some $R \geq T$. This yields the required estimate.

b) $\Rightarrow$ c). The definition of $\psi_{0}^{\prime}$ shows that $u_{0}^{\prime}(z) \leq H_{D}(z), z \in \mathbb{C}^{n}$. Then, by Lemma 9.1, there exists an entire function $\varphi$ of exponential type satisfying 1) and 2) in statement c) and having the property that for every $\varsigma \in \mathbb{S}$ there is a sequence $\left\{z_{k}(\varsigma)\right\} \in \Phi_{0}(\varsigma)$ with

$$
\lim _{k \rightarrow \infty}\left|z_{k}\right|^{-1}|\ln | \varphi\left(z_{k}(\varsigma)\right)\left|-u_{0}^{\prime}\left(z_{k}(\varsigma)\right)\right|=0 .
$$

By b) and the fact that $\left\{K_{m}\right\}$ is a sequence of compact sets exhausting $D$, it follows that

$$
\liminf _{k \rightarrow \infty}\left|z_{k}(\varsigma)\right|^{-1} \ln \left|\varphi\left(z_{k}(\varsigma)\right)\right| \geq H_{D}(\varsigma), \quad \varsigma \in \mathbb{S} \backslash \Xi .
$$

We show that similar sequences $\left\{z_{k}(\varsigma)\right\} \in \Phi_{0}(\varsigma)$ exist also for the points of $\partial(\mathbb{S} \backslash \Xi)$. Fix $\varsigma \in \partial(\mathbb{S} \backslash \Xi)$ and choose a sequence $\left\{\varsigma_{p}\right\} \subset \mathbb{S} \backslash \Xi$ convergent to $\varsigma$. The required sequence $\left\{z_{k}(\varsigma)\right\} \in \Phi_{0}(\varsigma)$ will be composed of points $z_{k}\left(\varsigma_{p}\right), p, k \geq 1$. By (10.7) and the definition of $\Phi_{0}(\varsigma)$, for every $p \geq 1$ there exists an index $k_{0}(p)$ such that for $k \geq k_{0}(p)$ we have

$$
\begin{aligned}
& \left|z_{k}\left(\varsigma_{p}\right)\right|^{-1} \ln \left|\varphi\left(z_{k}\left(\varsigma_{p}\right)\right)\right| \geq H_{D}\left(\varsigma_{p}\right)-1 / p \\
& \left|z_{k}\left(\varsigma_{p}\right)\right| \geq p, \quad\left|z_{k+1}\left(\varsigma_{p}\right)\right| /\left|z_{k}\left(\varsigma_{p}\right)\right| \leq 1+1 / p, \quad\left|z_{k}\left(\varsigma_{p}\right) /\right| z_{k}\left(\varsigma_{p}\right)\left|-\varsigma_{p}\right| \leq 1 / p .
\end{aligned}
$$

Next, we choose $k_{1}(p) \geq k_{0}(p)$ with

$$
\left|z_{k_{1}(p)}\left(\varsigma_{p}\right)\right| \geq\left|z_{k_{0}(p+1)}\left(\varsigma_{p+1}\right)\right| .
$$

We renumber all the points $z_{k_{0}(p)}\left(\varsigma_{p}\right), \ldots, z_{k_{1}(p)}\left(\varsigma_{p}\right)$ starting with $p=1$ in the natural order and denote the resulting sequence by $z_{1}(\varsigma), z_{2}(\varsigma), \ldots$; that is, we put $z_{1}(\varsigma)=$ $z_{k_{0}(1)}\left(\varsigma_{1}\right), \ldots, z_{k_{1}(1)-k_{0}(1)+1}(\varsigma)=z_{k_{1}(1)}\left(\varsigma_{1}\right), z_{k_{1}(1)-k_{0}(1)+2}(\varsigma)=z_{k_{0}(2)}\left(\varsigma_{2}\right)$, and so on. By $(10.9)$ and (10.10), the sequence $\left\{z_{k}(\varsigma)\right\}$ belongs to $\Phi_{0}(\varsigma)$. By (10.8) and the continuity of the function $H_{D}$, we have $\liminf _{k \rightarrow \infty}\left|z_{k}(\varsigma)\right|^{-1} \ln \left|\varphi\left(z_{k}(\varsigma)\right)\right| \geq H_{D}(\varsigma)$. From this and (10.7) it follows that $\underline{h}_{u_{0}^{\prime}}(\varsigma) \geq H_{D}(\varsigma)$ for all $\varsigma \in \overline{\mathbb{S} \backslash \Xi}$ (compare with the proof of the implication a) $\Rightarrow$ b) in Theorem 10.1). Since the lower indicator does not exceed the upper indicator, we obtain 3) in statement c), taking 2) into account.

The implication c) $\Rightarrow$ e) is obvious.

a) $\Rightarrow d$ ). Suppose a) is fulfilled. We apply Theorem 10.1 to see that c) in that theorem is true. By the definition of $\psi_{0}$ and $\psi_{p}$ and the fact that $\left\{K_{m}\right\}$ is an exhausting sequence of compact sets for $D$, it follows that $\underline{h}_{u_{0}}(\varsigma) \geq H_{D}(\varsigma), \varsigma \in \mathbb{S} \backslash \Xi$. By Lemma 2.7 in [30], this estimate shows that for every $\varsigma \in \mathbb{S} \backslash \Xi$ there exists a sequence $\left\{z_{k}(\varsigma)\right\} \in \Phi_{0}(\varsigma)$ with

$$
\liminf _{k \rightarrow \infty}\left|z_{k}(\varsigma)\right|^{-1}\left|u_{0}\left(z_{k}(\varsigma)\right)\right| \geq H_{D}(\varsigma)
$$

This leads to the required relations for $u_{0}$, as in the proof of $\left.\mathrm{b}\right) \Rightarrow \mathrm{c}$ ).

The implication $\mathrm{d}) \Rightarrow$ e) is obvious.

e) $\Rightarrow$ a). Suppose e) is fulfilled. It suffices to show that b) in Theorem 8.1 holds true. Fix an index $m$ and point $\varsigma \in \mathbb{S} \backslash \Xi$. Choose $\varepsilon>0$ such that

$$
H_{K_{m}}(z)+2 \varepsilon|z| \leq H_{D}(z), \quad z \in \mathbb{C}^{n} .
$$

Let $\varphi \in I_{W}$. Then $\ln |\varphi|=\ln \left|F_{W}\right|+\psi^{\prime}$, where $\psi^{\prime}$ is a plurisubharmonic function, and for some compact set $L \subset D$ and some constant $d>0$ we have

$$
\ln |\varphi(z)|=\ln \left|F_{W}(z)\right|+\psi^{\prime}(z) \leq d+H_{L}(z), \quad z \in \mathbb{C}^{n} .
$$


By Proposition 5.1, replacing $\psi^{\prime}$ if necessary with another plurisubharmonic function, we may assume that

$$
\ln \left|F_{W}(t \xi)\right|+\psi^{\prime}(t \xi) \geq-a_{0} t, \quad t \geq T,
$$

where $a_{0}, T$ are positive constants. Moreover, (10.12) will be preserved, possibly with another compact set $L \subset D$. We choose $\tau \in(0,1)$ such that

$$
\tau a_{0} \leq \varepsilon, \quad \tau b \leq \varepsilon,
$$

where $b=\sup _{|w|=1} H_{D}(w)$. Put

$$
\psi_{\varsigma, m}(z)=\tau \psi^{\prime}(z)+(1-\tau) \psi(z) .
$$

Then, by the definition of the upper indicator, formula (10.12), property 1) of $u$, and the fact that $L$ is a compact subset of $D$, we obtain

$$
h_{u_{\varsigma, m}}(z) \leq \tau h_{\varphi}(z)+(1-\tau) h_{u}(z)<H_{D}(z), \quad z \in \mathbb{C}^{n} \backslash\{0\},
$$

where $u_{\varsigma, m}=\ln \left|F_{W}\right|+\psi_{\varsigma, m}$. Next, by property 2 ) of $u$, formulas (10.11), (10.13), and the definition of the lower indicator, we have

$$
\underline{h}_{u_{\varsigma, m}}(\varsigma) \geq \tau \underline{h}_{\varphi}(\varsigma)+(1-\tau) \underline{h}_{u}(\varsigma) \geq-\varepsilon+H_{D}(\varsigma)-\varepsilon \geq H_{K_{m}}(\varsigma) .
$$

This completes the proof of the theorem.

In conclusion, we present yet another result about automatic analytic continuation. It says that if the functions in $W \subset H(D)$ extend to a convex domain $G$, then they extend automatically to a certain maximal convex domain constructed by $D$ and $G$ (this is the so-called $\Xi$-envelope of $D$ ).

Theorem 10.3. Let $D, G$ be convex domains with $D \subset G$ such that the set $\mathbb{S} \cap \Xi \backslash \operatorname{int} \Theta_{D}$ is closed. Next, let $W$ be a principal invariant subspace of $H(D)$ that admits spectral synthesis. Suppose that every function in $W$ admits analytic continuation to $G$ and can be approximated on $G$ by linear combinations of elements $E(W)$. Then every function in $W$ admits analytic continuation to $G(D)$ and can be approximated on $G(D)$ by linear combinations of elements $E(W)$.

Proof. Under the assumptions of the theorem, we can apply Theorem 10.1 to ensure statement b) in the statement of the latter. By Lemma 6.4 we have $\Xi(D, G)=\Xi(D, D(G))$. Therefore, statement a) in Theorem 10.1 holds true with $G(D)$ in the role of $G$.

Remark. In general, $G(D)$ differs from $G$. For example, consider an arbitrary triangle, and take the disk inscribed in it for $D$; for the role of $G$ we take an arbitrary convex domain including $D$, included in the triangle, and such that the boundary of $G$ intersects that of the disk only at the tangency points of the disk with the boundary of the triangle. Then $G(D)$ is the triangle itself, which, clearly, may fail to coincide with $G$.

\section{$\S 11$. Some EXAMPLES}

Theorems 8.1, 10.1, and 10.2 make it possible to easily construct examples of principal invariant subspaces $W \subset H(D)$ all functions in which extend to a larger convex domain $G$. For this, it suffices to take an arbitrary entire function of exponential type and regular growth. In this connection it should be noted that regular growth should occur practically for an arbitrary entire function of exponential type that is defined by a "finite formula" (i.e., via finitely many elementary functions), because construction of entire functions without regular growth on some subset of $\mathbb{C}^{n}$ is a fairly complicated procedure (though nowadays it is very well worked out). In most cases, this procedure involves existence theorems (in particular, the solvability of the $\bar{\partial}$-problem with estimates). 
So, let $f$ be an entire function of exponential type and regular growth. Suppose that its upper indicator $h_{f}$ is a convex function. Then it coincides with $H_{K}$ for some compact convex set $K \subset \mathbb{C}^{n}$. Let $D^{\prime}$ be an arbitrary convex domain in $\mathbb{C}^{n}$ such that the set $\mathbb{S} \cap \Xi \backslash \operatorname{int} \Theta_{D}$ is closed. We put $D=D^{\prime}+K$. If $f$ is not an exponential (which also forbids $f$ to be constant), it gives rise to a principal invariant subspace $W \subset H(D)$ admitting spectral synthesis. This subspace can be described in two ways. The first description is as follows. Let $N$ be the zero set of $f$ and $m(\lambda)$ the multiplicity of a zero $\lambda \in N$. Put $E=\left\{z^{\alpha} \exp \langle\lambda, z\rangle\right\}_{\lambda \in N, 0 \leq|\alpha|<m(\lambda)}$.

Then the required space $W \subset H(D)$ is the closure of the linear span of $E$. The set $N(W)$ coincides with $N$, and $f$ itself can play the role of $F_{W}$. The same subspace can be defined (see [6]) as the kernel of the convolution operator

$$
M[\varphi](z)=\mu(\varphi(z+y)), \quad \varphi \in H(D), \quad z \in D^{\prime},
$$

where $\mu$ is the functional in $H^{*}(D)$ whose Laplace transform is $f$ ( $f$ is the characteristic function of the convolution operator). It should be noted that, mostly, subspaces of precisely this type have been considered in earlier papers. We show that, in the situation in question, statement b) of Theorem 8.1 is true. Let $\left\{K_{m}^{\prime}\right\}$ be a sequence of compact convex sets exhausting $D^{\prime}$. Then $K_{m}=K_{m}^{\prime}+K, m=1,2, \ldots$, possesses the same property for $D$. Fix an index $m$. For every $\varsigma \in \mathbb{S}$, for the role of $\psi_{\varsigma, m}$ we take the support function of $K_{m}^{\prime}$ :

$$
\psi_{\varsigma, m}(z)=H_{K_{m}^{\prime}}(z), \quad z \in \mathbb{C}^{n} .
$$

Let $u_{\varsigma, m}=\ln \left|F_{W}\right|+\psi_{\varsigma, m}=\ln |f|+\psi_{\varsigma, m}$. Since $f$ is of regular growth, the addition theorem for the indicators of plurisubharmonic functions (see [37]) shows that

$$
h_{u_{\varsigma, m}}(z)=h_{\ln \left|F_{W}\right|}(z)+h_{\psi_{\varsigma, m}}(z)=h_{f}(z)+h_{\psi_{\varsigma, m}}(z), \quad z \in \mathbb{C}^{n} .
$$

By the definition of $K$ and $\psi_{\varsigma, m}$, it follows that

$$
h_{u_{\varsigma, m}}(z)=H_{K}(z)+H_{K_{m}^{\prime}}(z)=H_{K_{m}}(z)<H_{D}(z), \quad z \in \mathbb{C}^{n} \backslash\{0\} .
$$

The function $u_{\varsigma, m}$ is of regular growth because it is the sum of $\ln |f|$ and $H_{K_{m}}$, both being of regular growth (for $H_{K_{m}}$ this property can easily be verified). Therefore,

$$
\underline{h}_{u_{\varsigma, m}}(z)=h_{u_{\varsigma, m}}(z), \quad z \in \mathbb{C}^{n} .
$$

By the above,

$$
\underline{h}_{u_{\varsigma, m}}(z)=H_{K_{m}}(z), \quad z \in \mathbb{C}^{n} .
$$

Thus, statement b) in Theorem 8.1 is true, whence a) is also true. In particular, every function in $W$ extends analytically to any subdomain $G$ of $D(W)$ that includes $D$.

Now, take an arbitrary polynomial $p(z)$ for the role of $f(z)$. Then $f(z)=p(z)$ is of minimal type; i.e., for every $\varepsilon>0$ we have

$$
|p(z)| \leq c(\varepsilon) \exp (\varepsilon|z|), \quad c(\varepsilon)>0, \quad z \in \mathbb{C}^{n} .
$$

It follows that $h_{p}(z)=0, z \in \mathbb{C}^{n}$. Being a function of minimal type, $p(z)$ is of regular growth (see [37]), i.e., $\underline{h}_{p}(z)=h_{p}(z), z \in \mathbb{C}^{n}$. Thus, we are in the situation described above. However, $h_{p}(z) \equiv 0$ in this case, whence we see that $K$ coincides with the origin (its support function is identically zero). The space $W \subset H(D)=H\left(D^{\prime}\right)$ turns into the space of solutions of a homogeneous partial differential equation with constant coefficients (the characteristic function of this equation coincides with $p(z)$ ). By the above, all such solutions analytic in the convex domain $D=D^{\prime}+K=D^{\prime}$ extend to $D(W)$. This is a result of [27.

Now let $f(z)$ be an arbitrary entire function of exponentially minimal type. Then $\underline{h}_{p}(z)=h_{p}(z)=0, z \in \mathbb{C}^{n}$. As before, every function in $W \subset H(D)$ (in our case, $W$ is the space of solutions of a homogeneous partial differential equation of infinite order 
with constant coefficients) extends analytically to $D(W)$. This yields the result of 28 ] (and even more, because no restrictions except that of being of minimal type are now imposed on $f$ ).

In a similar manner, we can obtain continuability results from other papers by predecessors. For instance, the results of [8] and [29] are easy consequences of Theorem 4.1 (and, in fact, they look much like that theorem).

Now, we consider a specific example of a principal invariant subspace. Let $D$ be a convex domain in $\mathbb{C}^{2}$, and let

$$
N=\left\{\left(\lambda_{1}, \lambda_{2}\right) \in \mathbb{C}^{2}: \lambda_{2}=\pi k, k=1,2, \ldots\right\}, \quad E=\{\exp \langle\lambda, z\rangle, \lambda \in N\} .
$$

For $W$ we take the closure of the linear hull of $E$ in the topology of $H(D)$. The set $N$ is included in the zero set of the function $f(\lambda)=\sin \lambda_{2}$. This is a function of exponential type and regular growth whose upper indicator is $h_{f}(\lambda)=\left|\operatorname{Im} \lambda_{2}\right|, \lambda \in \mathbb{C}^{2}$. This indicator coincides with the support function of an interval on the imaginary axis in the $\lambda_{2}$-plane: $K=\left\{\left(0, i y_{2}\right) \in \mathbb{C}^{2}: y_{2} \in[-1,1]\right\}$. If $D$ includes a translate of this interval, the subspace $W \subset H(D)$ is nontrivial and principal. In this case, $N=N(W)$ and $W$ is not the space of solutions of any homogeneous convolution equation (however, for special domains $D$, it is the space of solutions of a system of two convolution equations with appropriate characteristic functions; see [38, Theorem 7.5]). The role of $F_{W}$ can be played by the part of the infinite product expansion for the sine involving only positive zeros. The function $F_{W}$ is of the first order of growth and of infinite type. We denote by $F$ the entire function such that

$$
f(\lambda)=\sin \lambda_{2}=F_{W}(\lambda) F(\lambda), \quad \lambda \in \mathbb{C}^{2} .
$$

Let $D^{\prime}$ be an arbitrary convex domain in $\mathbb{C}^{2}$ with the property that the set $\mathbb{S} \cap \Xi \backslash$ int $\Theta_{D}$ is closed. We put $D=D^{\prime}+K$ and consider the same sequences $\left\{K_{m}^{\prime}\right\},\left\{K_{m}\right\}$ of compact sets as above. We show that in this case statement b) of Theorem 8.1 is true for $D$. Fix an index $m$. For every $\varsigma \in \mathbb{S}$, put

$$
\psi_{\varsigma, m}(\lambda)=\ln |F(\lambda)|+H_{K_{m}^{\prime}}(\lambda), \quad \lambda \in \mathbb{C}^{2} .
$$

As above, it can be proved that the function

$$
u_{\varsigma, m}=\ln \left|F_{W}\right|+\psi_{\varsigma, m}=\ln \left|F_{W}\right|+\ln |F|+H_{K_{m}^{\prime}}=\ln |f|+H_{K_{m}^{\prime}}
$$

obeys the inequalities

$$
h_{u_{\varsigma, m}}(\lambda)<H_{D}(\lambda), \quad \lambda \in \mathbb{C}^{2} \backslash\{0\}, \quad \underline{h}_{u_{\varsigma, m}}(\lambda) \geq H_{K_{m}}(\lambda), \quad \lambda \in \mathbb{C}^{2} .
$$

By Theorem 8.1, every function in $W$ extends analytically to $D(W)$. For example, if $D^{\prime}$ is the ball of radius 1 and centered at zero, then

$$
D(W)=\left\{z \in \mathbb{C}^{2}: \operatorname{Re}\left(\lambda_{1} z_{1}+t_{2} z_{2}\right)<1,\left|\lambda_{1}\right|^{2}+t_{2}^{2}=1, t_{2} \geq 0\right\} .
$$

The domain $D(W)$ is the cylinder

$$
D(W)=\left\{\left(z_{1}, x_{2}, y_{2}\right) \in \mathbb{C}^{2}:\left(z_{1}, x_{2}\right) \in B, y_{2} \in \mathbb{R}\right\},
$$

where $B \subset \mathbb{R}^{3}$ is the union of the half-cylinder $\left\{\left(z_{1}, x_{2}\right):\left|z_{1}\right|<1, x_{2} \leq 0\right\}$ and the half-ball $\left\{\left(z_{1}, x_{2}\right):\left|z_{1}\right|^{2}+x_{2}^{2}<1, x_{2} \geq 0\right\}$.

Consider yet another function, namely, $f(\lambda)=\cos \sqrt{\lambda_{1} \lambda_{2}}, \lambda \in \mathbb{C}^{2}$. It is entire and of exponential type and regular growth; its upper indicator is $h_{f}(\lambda)=\left|\operatorname{Im} \sqrt{\lambda_{1} \lambda_{2}}\right|$, $\lambda \in \mathbb{C}^{2}$. But in distinction to the previous cases, $h_{f}(\lambda)$ is not a convex function (it vanishes at the coordinate planes). Therefore, it does not coincide with the support function of any compact set. Let $D$ be a bounded strictly convex domain with smooth boundary, and suppose that $D$ contains a translate of the polydisk $U=\left\{\left(\lambda_{1}, \lambda_{2}\right) \in \mathbb{C}^{2}\right.$ : $\left.\left|\lambda_{1}\right| \leq 1,\left|\lambda_{2}\right| \leq 1\right\}$. 
Then $f(\lambda)$ determines a nontrivial closed principal invariant subspace $W \subset H(D)$, which is the space of solutions of the homogeneous convolution equation whose characteristic function is $f(\lambda)$. We can take $f$ for the role of $F_{W}$. Suppose that every function in $W$ extends analytically to $D(W)$ and admits an approximation on $D(W)$ by a linear combination of elements of $E(W)$. By Theorem 10.2, there exists an entire function $\varphi$ of exponential type such that $g=\varphi / f$ is an entire function and

$$
h_{\varphi}(\varsigma)=H_{D}(\varsigma), \quad \varsigma \in \overline{\mathbb{S} \backslash \Xi} .
$$

We show that in our case the sets $\Xi \cap \mathbb{S}$ and $\Delta(W)$ coincide. By (3.1) and the definition of $\Xi$ we see that $\Delta(W) \subset \Xi \cap \mathbb{S}$. Let $\lambda \in \Xi \cap \mathbb{S}=\Xi(D, D(W)) \cap \mathbb{S}$. Then $H_{D}(\lambda)=H_{D(W)}(\lambda)$. Using the definition of the support function, we find $z \in \bar{D}$ with $\operatorname{Re}\langle z, \lambda\rangle=H_{D}(\lambda)$. This formula shows that $z$ belongs to the support hyperplane $\{w \in$ $\left.\mathbb{C}^{2}: \operatorname{Re}\langle w, \lambda\rangle=H_{D}(\lambda)\right\}$ for $D$. If $z \in D(W)$, then $H_{D(W)}(\lambda)>\operatorname{Re}\langle z, \lambda\rangle$, whence $H_{D(W)}>H_{D}(\lambda)$, contradicting the choice of $\lambda$. Therefore, $z \notin D(W)$. By the definition of $D(W)$, there exists $\xi \in \Delta(W)$ such that $\operatorname{Re}\langle z, \xi\rangle \geq H_{D(W)}(\xi)$. On the other hand, $\operatorname{Re}\langle z, \xi\rangle \leq H_{D}(\xi) \leq H_{D(W)}(\xi)$ because $z \in \bar{D}$ and $D \subset D(W)$. Thus, $\operatorname{Re}\langle z, \xi\rangle=H_{D}(\xi)$; i.e., $z$ lies on the support hyperplane $\left\{w \in \mathbb{C}^{2}: \operatorname{Re}\langle w, \xi\rangle=H_{D}(\xi)\right\}$ for $D$. Recall that $D$ has been chosen to be smooth. Therefore, only one support hyperplane passes through each boundary point of it (in particular, through $z$ ). We see that $\lambda$ and $\xi$ coincide, i.e., $\lambda \in \Delta(W)$. So, $\Xi \cap \mathbb{S}=\Delta(W)$. By (11.1) it follows that

$$
h_{\varphi}(\varsigma)=H_{D}(\varsigma), \quad \varsigma \in \overline{\mathbb{S} \backslash \Delta(W)} .
$$

Since $f$ is of regular growth, the addition theorem for indicators yields $h_{\varphi}(\lambda)=h_{f}(\lambda)+$ $h_{g}(\lambda), \lambda \in \mathbb{C}^{2}$. Taking the above into account, we arrive at

$$
h_{f}(\varsigma)+h_{g}(\varsigma)=H_{D}(\varsigma), \quad \varsigma \in \overline{\mathbb{S} \backslash \Delta(W)} .
$$

Fix $\lambda \in \mathbb{S}$ with $\lambda_{1} \neq 0$ and $\lambda_{2} \neq 0$. The functions $h_{f, g}(\alpha)=h_{f}(\alpha \lambda)$ and $h_{g, \lambda}(\alpha)=$ $h_{g}(\alpha \lambda), \alpha \in \mathbb{C}$, are positive homogeneous of degree 1 and subharmonic. Consequently, they are convex, and they determine compact convex sets $L$ and $L^{\prime}$ on the complex plane such that $H_{L}(\alpha)=h_{f, \lambda}(\alpha)$ and $H_{L^{\prime}}(\alpha)=h_{g, \lambda}(\alpha), \alpha \in \mathbb{C}$. In its turn, the function $H_{D}(\alpha \lambda), \alpha \in \mathbb{C}$, determines a convex planar domain $D(\lambda): H_{D(\lambda)}(\alpha)=H_{D}(\alpha \lambda), \alpha \in \mathbb{C}$. By (11.2), we have

$$
H_{L}(\alpha)+H_{L^{\prime}}(\alpha)=H_{D(\lambda)}(\alpha), \quad \alpha \lambda \in \overline{\mathbb{S} \backslash \Delta(W)} .
$$

We show that, here, any number of the form $e^{i \tau}$ with $\tau \in[0,2 \pi]$ can play the role of $\alpha$.

The set $N(W)$ coincides with the zero set of $f(\lambda)$; i.e.,

$$
N(W)=\left\{\left(\xi_{1}, \xi_{2}\right) \in \mathbb{C}^{2}: \xi_{1} \xi_{2}=(\pi / 2+\pi k)^{2}, k=0,1, \ldots\right\} .
$$

Therefore, $N(W)$ and (with it) $\Delta(W)$ lie in the cone

$$
\Gamma=\left\{\left(\xi_{1}, \xi_{2}\right) \in \mathbb{C}^{2}: \xi_{1} \xi_{2}>0\right\} .
$$

The complex line $\alpha \lambda, \alpha \in \mathbb{C}$, intersects $\Gamma$ by the real line $t e^{i \tau_{0}} \lambda, t \in \mathbb{R}$, for some $\tau_{0} \in[0, \pi)$. This means that (11.3) is true for all $\alpha=e^{i \tau}, \tau \in[0,2 \pi]$. Since the functions in that formula are positive homogeneous, we obtain

$$
H_{L}(\alpha)+H_{L^{\prime}}(\alpha)=H_{D(\lambda)}(\alpha), \quad \alpha \in \mathbb{C} .
$$

It follows that $\overline{D(\lambda)}$ is the algebraic sum $L+L^{\prime}$. The compact set $L$ is an interval. Therefore, the boundary of $D(\lambda)$ includes two intervals parallel to $L$. Since $D(\lambda)$ is a projection of $D$, we see that $D$ also has intervals on the boundary. But this is impossible because $D$ is a strictly convex domain. 
Now let $D$ be the unbounded domain equal to the algebraic sum of the complex hyperplane $T=\left\{z \in \mathbb{C}^{2}: z_{1}+z_{2}=0\right\}$ and the following subset $\Pi$ of the complex line $\beta(1,1), \alpha \in \mathbb{C}:$

$$
\Pi=\left\{z \in \mathbb{C}^{2}: z=\beta(1,1), \operatorname{Re} \beta<0,|\operatorname{Im} \beta|<1\right\} .
$$

It is easy to observe that $\Theta_{D}$ coincides with the open set

$$
\mathbb{C}^{2} \backslash\left\{\lambda \in \mathbb{C}^{2}: \lambda=\alpha(1,1), \operatorname{Re} \alpha \geq 0\right\}=\mathbb{C}^{2} \backslash \Sigma
$$

and $H_{D}(\lambda)=2|\operatorname{Im} \alpha|, \lambda=\alpha(1,1) \in \Sigma$.

The function $f(\lambda)=\cos \sqrt{\lambda_{1} \lambda_{2}}$ determines a nontrivial subspace $W$ of $H(D)$ (because $I_{W}$ is nontrivial since it contains the functions $\left.f(\lambda) \exp \left(-\varepsilon\left(\lambda_{1}+\lambda_{2}\right)\right), \varepsilon>0\right)$. It can easily be observed that now $D(W)$ is a half-space: $D(W)=\left\{z \in \mathbb{C}^{2}: \operatorname{Re}\left(z_{1}+z_{2}\right)<0\right\}$; also, the set $\mathbb{S} \cap \Xi \backslash \operatorname{int} \Theta_{D}=\left\{(\sqrt{2})^{-1}(1,1)\right\}$ is closed. We put

$$
\psi_{\varsigma, m}(\lambda)=(1-\delta)\left|\operatorname{Im} \sqrt{\lambda_{1} \lambda_{2}}\right|-\delta \operatorname{Re}\left(\lambda_{1}+\lambda_{2}\right), \quad \delta>0 .
$$

Then

$$
\begin{aligned}
h_{u_{\varsigma, m}}(\lambda) & <H_{D}(\lambda), \quad \lambda \in \mathbb{C}^{2} \backslash\{0\}, \\
\underline{h}_{u_{\varsigma, m}}(\lambda) & \geq H_{D}(\lambda)-3 \delta|\alpha|, \quad \lambda=\alpha(1,1) \in \Sigma,
\end{aligned}
$$

where $u_{\varsigma, m}=\ln \left|F_{W}\right|+\psi_{\varsigma, m}=\ln |f|+\psi_{\varsigma, m}$. This means that statement b) in Theorem 8.1 holds true. By that theorem, every function in $W$ admits analytic continuation to the half-space $D(W)$.

Finally, consider the domain

$$
D=\left\{z \in \mathbb{C}^{2}: \operatorname{Re}\left(i\left(z_{1}+z_{2}\right)\right)<0\right\} .
$$

As above, the function $f(\lambda)=\cos \sqrt{\lambda_{1} \lambda_{2}}$ determines a nontrivial subspace $W$ in $H(D)$. Moreover, $\Delta(W) \subset$ int $\Theta_{D}$. By Corollary 8.1, every function in $W$ extends up to an entire function.

\section{REFERENCES}

[1] V. V. Napalkov, Convolution equations in multidimensional spaces, Nauka, Moscow, 1982. (Russian) MR0678923 (86g:46054)

[2] J. Hadamard, Essai sur l'étude des fonctions données par leur développement de Taylor, J. Math. Pures Appl. Ser. 48 (1892), 101-106.

[3] E. Fabry, Sur les points singuliers d'une fonction donnée par son développement de Taylor, Ann. École Norm. Sup. (3) 13 (1896), 367-399. MR.1508933

[4] I. F. Krasichkov-Ternovskiŭ, Invariant subspaces of analytic functions. I. Spectral synthesis on convex domains, Mat. Sb. (N.S.) 87 (1972), no. 4, 459-489; English transl., Math. USSR-Sb. 16 (1972), no. 4, 471-500. MR0422636(54:10622)

[5] _ Invariant subspaces of analytic functions. II. Spectral synthesis on convex domains, Mat. Sb. (N.S.) 88 (1972), no. 1, 3-30; English transl., Math. USSR-Sb. 17 (1972), no. 1, 1-29. MR0422636 (54:10622)

[6] R. S. Yulmukhametov, Homogeneous convolution equations, Dokl. Akad. Nauk SSSR 316 (1991), no. 2, 312-315; English transl., Soviet Math. Dokl. 43 (1991), no. 1, 101-103. MR1100598 (92c:32005)

[7] A. S. Krivosheev and V. V. Napalkov, Complex analysis and convolution operators, Uspekhi Mat. Nauk 47 (1992), no. 6, 3-58; English transl., Russian Math. Surveys 47 (1992), no. 6, 1-56. MR 1209144 (94e:32003)

[8] I. F. Krasichkov-Ternovskiı̌, Spectral synthesis and analytic continuation, Uspekhi Mat. Nauk 58 (2003), no. 1, 33-112; English transl., Russian Math. Surveys 58 (2003), no. 1, 31-108. MR1992131 (2004f:46037)

[9] G. Polya, Uber die Exstenz unendlich vieler singularer Punkte auf der Konvergenzgeraden gewisser Dirichlet'sher Reihen, Sitzungber. Preu. Akad. Wiss. 1923, 45-50.

[10] _ Eine Verallgemeinerung des Fabryschen Lückensatzes, Nachr. Ges. Wiss. Gottingen, Math.-Phys. Kl. 2 (1927), 187-195. 
[11] V. Bernstein, Leçons sur les progress de la théorie des series de Dirichlet, Gauthier-Villars, Paris, 1933.

[12] A. Ostrowski, Uber die analytische Fortsetzung von Taylorschen und Dirichletschen Reihen, Math. Ann. 129 (1955), 1-43. MR.0069878 (16:1094a)

[13] A. F. Leont'ev, On a class of functions defined by series of Dirichlet polynomials, Uspekhi Mat. Nauk 3 (1948), no. 4, 176-180. (Russian) MR0027854 (10:364a)

[14] _ Series of Dirichlet polynomials and their generalizations, Trudy Mat. Inst. Steklov. 39 (1951), 1-215. (Russian) MR0055444 (14:1074e)

[15] J.-P. Kahane, Sur quelques problèmes d'unicité et de prolongement, relatifs aux fonctions approchables par des sommes d'exponentielles, Ann. Inst. Fourier (Grenoble) 5 (1953-1954), 39-130 (1955). MR0075350 (17:732b)

[16] A. F. Leont'ev, On convergence of a sequence of Dirichlet polynomials, Dokl. Akad. Nauk SSSR 108 (1956), no. 1, 23-26. (Russian) MR.0080180 (18:205d)

[17] _ New proof of a theorem on convergence of a sequence of Dirichlet polynomials, Uspekhi Mat. Nauk 12 (1957), no. 3, 165-170. (Russian) MR0089268(19:643e)

[18] _ Properties of sequences of Dirichlet polynomials which are convergent on an interval of the imaginary axis, Izv. Akad. Nauk SSSR Ser. Mat. 29 (1965), no. 2, 269-328. (Russian) MR0180809 (31:5039)

[19] L. Schwartz, Étude des sommes d'exponentielles, Hermann, Paris, 1959. MR0106383 (21:5116)

[20] A. Baillette, Approximation de fonctions par des sommes d'exponentielles, C. R. Acad. Sci. Paris 249 (1959), 2470-2471. MR0117351 (22:8131)

[21] _ Fonctions approchables par des sommes d'exponentielles, J. Anal. Math. 10 (1962-1963), 91-115. MR0149168 (26:6660)

[22] I. F. Krasichkov-Ternovskiŭ, On convergence of Dirichlet polynomials, Sibirsk. Mat. Zh. 7 (1966), no. 5, 1039-1058. (Russian) MR0210879 (35:1764)

[23] _ Invariant subspaces of analytic functions. Analytic continuation, Izv. Akad. Nauk SSSR Ser. Mat. 37 (1973), no. 4, 931-945; English transl., Math. USSR-Izv. 7 (1973), 933-947. MR0367233(51:3475)

[24] A. S. Krivosheev, Analytic continuation of a function from invariant subspaces in convex domains of a complex space, Izv. Ross. Akad. Nauk Ser. Mat. 62 (1998), no. 2, 75-102; English transl., Izv. Math. 62 (1998), no. 2, 287-312. MR1623826 (2000d:32019)

[25] _ Analytic continuation of functions from invariant subspaces, Dokl. Akad. Nauk 386 (2002), no. 4, 450-452. (Russian) MR2006037

[26] _ A criterion for the analytic continuation of functional belonging to invariant subspaces in convex domains of the complex plane, Izv. Ross. Akad. Nauk Ser. Mat. 68 (2004), no. 1, 43-78; English transl., Izv. Math. 68 (2004), no. 1, 43-76. MR.2096937(2005g:30003)

[27] C. O. Kiselman, Prolongement des solutions d'une équation aux dérivées partielles à coefficients constants, Bull. Soc. Math. France 97 (1969), 329-356. MR.0267259 (42:2161)

[28] A. Sebbar, Prolongement des solutions holomorphes de certains opérateurs différentiels d'ordre infini à coefficients constants, Lecture Notes in Math., vol. 822, Springer, Berlin, 1980, pp. 199220. MR0599028 (82f:35049)

[29] A. Meril and D. C. Struppa, Convolutors in spaces of holomorphic functions, Lecture Notes in Math., vol. 1276, Springer, Berlin, 1987, pp. 253-275. MR0922324 (89e:32003)

[30] A. S. Krivosheev, Indicators of entire functions and the continuation of solutions of a homogeneous convolution equation, Mat. Sb. 184 (1993), no. 8, 81-108; English transl., Russian Acad. Sci. Sb. Math. 79 (1994), no. 2, 401-423. MR1239760 (94k:32002)

[31] R. Ishimura and Y. Okada, The existence and the continuation of holomorphic solutions for convolution equations in tube domains, Bull. Soc. Math. France 122 (1994), 413-433. MR.1294464 (96f:32013)

[32] Von K. Leichtweiss, Konvexe Mengen, Springer-Verlag, Berlin-New York, $1980 . \quad$ MR0586235 (81j:52001)

[33] L. I. Ronkin, Introduction to the theory of entire functions of several variables, Nauka, Moscow, 1971; English transl., Transl. Math. Monogr., vol. 44, Amer. Math. Soc., Providence, RI, 1974. MR0320357 (47:8896) MR0346175 (49:10901)

[34] R. E. Edwards, Functional analysis. Theory and applications, Holt, Rinehart and Winston, New York, 1965. MR 0221256 (36:4308)

[35] A. Grothendieck, Sur les espaces $(F)$ et (DF), Summa Bras. Math. 3 (1954), 57-123. MR0075542 (17:765b) 
[36] L. Hörmander, The analysis of linear partial differential operators. I. Distribution theory and Fourier analysis, Grundlehren Math. Wiss., Bd. 256, Springer-Verlag, Berlin, 1983. MR0717035 (85g:35002a)

[37] P. Lelong and L. Gruman, Entire functions of several complex variables, Grundlehren Math. Wiss., Bd. 282, Springer-Verlag, Berlin, 1986. MR.0837659 (87j:32001)

[38] A. F. Leont'ev, Entire functions. Series of exponentials, Nauka, Moscow, 1983. (Russian) MR 0753827 (86j:30005)

[39] R. S. Yulmukhametov, Entire functions of several variables with given behavior at infinity, Izv. Ross. Akad. Nauk Ser. Mat. 60 (1996), no. 4, 205-224; English transl., Izv. Math. 60 (1996), no. 4, 857-879. MR 1416928 (98a:32003)

[40] B. Ya. Levin, Distribution of zeros of entire functions, Gostekhizdat, Moscow, 1956; English transl., Transl. Math. Monogr., vol. 5, Amer. Math. Soc., Providence, RI, 1980. MR0087740 (19:402c) MR.0589888(81k:30011)

Mathematical Institute with Computer Center, Urals Scientific Center, Russian Academy of Sciences, Ul. Chernyshevskogo 112, Ufa 450077, Russia

E-mail address: sasha@matem.anrb.ru

Received 1/APR/2009

Translated by the AUTHOR 